\title{
Synthesis of Enantioenriched Propargylic Alcohols Related to Polyketide Natural Products. A Comparison of Methodologies
}

James A. Marshall* and Matthew P. Bourbeau

Department of Chemistry, P. O Box 400319 University of Virginia

Charlottesville Virginia 22904

Supporting Information

Experimental procedures for all intermediates and selected ${ }^{1} \mathrm{H}$ NMR spectra. 
<smiles>[R5]OCC(C)C(O)C#CC#N</smiles>

(3R, 4R)-5-(t-Butyldimethylsilyloxy)-4-methyl-1-trimethylsilyl-1-pentyn-3-ol (2a). A. Addition with (S)-BINOL as the Chiral Ligand.

The standard procedure for TMS acetylene addition was employed (see below) using aldehyde 1a (0.10 g, $0.49 \mathrm{mmol})$, TMS acetylene $(0.28 \mathrm{~mL}, 2.0 \mathrm{mmol}), \mathrm{Et}_{2} \mathrm{Zn}(0.20 \mathrm{~mL}, 2.0 \mathrm{mmol})$, Ti(OiPr $)_{4}(0.15 \mathrm{~mL}$, $0.49 \mathrm{mmol})$, and $(S)$-BINOL (0.056 g, $0.20 \mathrm{mmol})$, affording alcohol $2 \mathrm{a}(0.11 \mathrm{~g}, 73 \%$ yield, $10: 1 \mathrm{mixture}$ of diastereomers) as a clear oil. $\mathrm{R}_{\mathrm{f}}=0.3(10 \%$ EtOAc/ hexanes $) ;[\alpha]_{20}^{\mathrm{D}}=-6.0\left(\mathrm{c}=1.47, \mathrm{CHCl}_{3}\right) ; \mathrm{IR}($ film) 3432, $2174 \mathrm{~cm}^{-1} ;{ }^{1} \mathrm{H} \mathrm{NMR}\left(\mathrm{CDCl}_{3}\right.$, major diastereomer) $\delta 4.39(\mathrm{t}, \mathrm{J}=5.5 \mathrm{~Hz}, 1 \mathrm{H}), 3.93(\mathrm{dd}, \mathrm{J}=4.0 \mathrm{~Hz}$, $10.0 \mathrm{~Hz}, 1 \mathrm{H}), 3.57(\mathrm{dd}, \mathrm{J}=6.5 \mathrm{~Hz}, 10.0 \mathrm{~Hz}, 1 \mathrm{H}), 3.43(\mathrm{~d}, \mathrm{~J}=5.5 \mathrm{~Hz}, 1 \mathrm{H}), 1.96-1.88(\mathrm{~m}, 1 \mathrm{H}), 1.01(\mathrm{~d}, \mathrm{~J}=$ $7.0 \mathrm{~Hz}, 3 \mathrm{H}), 0.90(\mathrm{~s}, 9 \mathrm{H}), 0.17(\mathrm{~s}, 9 \mathrm{H}), 0.08(\mathrm{~s}, 3 \mathrm{H}), 0.08(\mathrm{~s}, 3 \mathrm{H}) ;{ }^{13} \mathrm{C} \mathrm{NMR}\left(\mathrm{CDCl}_{3}\right) \delta 105.72,89.76,67.14$, 66.78, 40.44, 25.81, 18.15, 12.91, -0.12, -5.63, -5.66; Anal. Calcd for $\mathrm{C}_{15} \mathrm{H}_{32} \mathrm{O}_{2} \mathrm{Si}_{2}$ : C, 59.94, H, 10.73, Found: C, 59.97, H, 10.54 .

\section{B. Without BINOL}

The standard procedure for TMS acetylene addition was employed using aldehyde $1 \mathbf{a}(0.10 \mathrm{~g}, 0.49$ mmol), TMS acetylene $(0.28 \mathrm{~mL}, 2.0 \mathrm{mmol}), \mathrm{Et}_{2} \mathrm{Zn}(0.20 \mathrm{~mL}, 2.0 \mathrm{mmol})$, and Ti(OiPr $)_{4}(0.15 \mathrm{~mL}, 0.49$ mmol), affording alcohol $3 \mathrm{a}(0.072 \mathrm{~g}, 48 \%$ yield, 1.5:1 mixture of diastereomers favoring the $3 R$ diastereomer) as a clear oil. All analytical data was consistent with the BINOL directed cases.

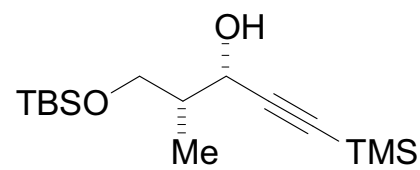

(3S, 4R)-5-(t-Butyldimethylsilyloxy)-4-methyl-1-trimethylsilyl-1-pentyn-3-ol (3a)

\section{Standard Procedure for TMS Acetylide Addition to Aldehydes}

TMS acetylene $(0.28 \mathrm{~mL}, 2.7 \mathrm{mmol})$ was taken up in $2 \mathrm{~mL}$ of toluene. $\mathrm{Et}_{2} \mathrm{Zn}(0.20 \mathrm{~mL}, 2.0 \mathrm{mmol})$ was added to the solution carefully. The mixture was heated to reflux for $1 \mathrm{~h}$, during which time a large amount of grey precipitate formed in the reaction flask. The mixture was cooled to room temperature, and $(R)$-BINOL $(0.056 \mathrm{~g}, 0.20 \mathrm{mmol}), \mathrm{Et}_{2} \mathrm{O}(8 \mathrm{~mL})$, and $\mathrm{Ti}(\mathrm{OiPr})_{4}(0.15 \mathrm{~mL}, 0.49 \mathrm{mmol})$ were added. After 1 h, aldehyde 2a $(0.050 \mathrm{~mL}, 0.42 \mathrm{mmol})$ was added, and the mixture was stirred overnight. The reaction was quenched with 1.0 $\mathrm{M}$ tartaric acid and the mixture was stirred for $30 \mathrm{~min}$. The mixture was partitioned in a separatory funnel, and the aqueous portion was extracted three times with $\mathrm{Et}_{2} \mathrm{O}$. The combined organic extracts were washed with brine and dried over $\mathrm{MgSO}_{4}$. Filtration and concentration, followed by flash chromatography on silica gel (2.5\% EtOAc/ hexanes to 5\% EtOAc/ hexanes gradient), afforded propargylic alcohol 3a $(0.10 \mathrm{~g}, 67 \%$ yield $)$ as a light yellow oil. $\mathrm{R}_{\mathrm{f}}=0.3(10 \%$ EtOAc/ hexanes $) ;[\alpha]_{20}^{\mathrm{D}}=-24.1(\mathrm{c}=$ $\left.1.21, \mathrm{CHCl}_{3}\right)$; IR (film) 3441, $2183 \mathrm{~cm}^{-1} ;{ }^{1} \mathrm{H} \mathrm{NMR}\left(\mathrm{CDCl}_{3}\right.$, major diastereomer $) \delta 4.42(\mathrm{dd}, \mathrm{J}=3.5 \mathrm{~Hz}, 8.0$ 
$\mathrm{Hz}, 1 \mathrm{H}), 3.93(\mathrm{~d}, \mathrm{~J}=7.5 \mathrm{~Hz}, 1 \mathrm{H}), 3.86(\mathrm{t}, \mathrm{J}=9.5 \mathrm{~Hz}, 1 \mathrm{H}), 3.67(\mathrm{dd}, \mathrm{J}=4.5 \mathrm{~Hz}, 9.5 \mathrm{~Hz}, 1 \mathrm{H}), 2.18-2.11(\mathrm{~m}$, $1 \mathrm{H}), 0.91(\mathrm{~s}, 9 \mathrm{H}), 0.88(\mathrm{~d}, \mathrm{~J}=7.0 \mathrm{~Hz}, 3 \mathrm{H}), 0.18 \mathrm{9s}, 9 \mathrm{H}), 0.11(\mathrm{~s}, 3 \mathrm{H}), 0.09(\mathrm{~s}, 3 \mathrm{H}) ;{ }^{13} \mathrm{C} \mathrm{NMR}\left(\mathrm{CDCl}_{3}\right) \delta$ $104.91,90.08,67.62,66.73,39.59,25.82,18.14,12.48,-0.08,-5.58,-5.72$.

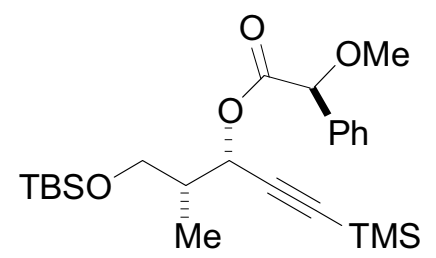

\section{(S)-O-Methyl Mandelic Ester of 3a}

Alcohol 3a $(0.010 \mathrm{~g}, 0.033 \mathrm{mmol})$ was taken up in $1 \mathrm{~mL}$ of $\mathrm{CH}_{2} \mathrm{Cl}_{2}$. (S)-O-methyl mandelic acid $(0.011 \mathrm{~g}, 0.066 \mathrm{mmol})$, DCC $(0.014 \mathrm{~g}, 0.066 \mathrm{mmol})$, and a few crystals of DMAP were added, and the mixture was stirred for $15 \mathrm{~min}$. The solvent was removed under vacuum, and the residue was purified by flash chromatography on silica gel (100\% hexanes), affording the desired ester $(0.008 \mathrm{~g}, 53 \%)$ yield as a clear oil. ${ }^{1} \mathrm{H}$ NMR $\left(\mathrm{CDCl}_{3}\right)$ 8 7.47-7.26 (m, 5H), $5.46(\mathrm{~d}, \mathrm{~J}=5.4 \mathrm{~Hz}, 1 \mathrm{H}), 4.79(\mathrm{~s}, 1 \mathrm{H}), 3.56-3.43(\mathrm{~m}, 2 \mathrm{H})$, 3.45 (s, 3H), 2.05-1.95 (m, 1H), $0.96(\mathrm{~d}, \mathrm{~J}=6.9 \mathrm{~Hz}, 3 \mathrm{H}), 0.86(\mathrm{~s}, 9 \mathrm{H}), 0.08$ (s, 9H), -0.01 (s, 3H), -0.03 (s, $3 \mathrm{H})$.<smiles>C#CC#C[C@@H](OC(=O)C(OC)c1ccccc1)C(C)CO[R5](F)(F)c1ccccc1</smiles>

\section{(R)-O-Methyl Mandelic Ester of 3a}

Alcohol 3a $(0.010 \mathrm{~g}, 0.033 \mathrm{mmol})$ was taken up in $1 \mathrm{~mL}$ of $\mathrm{CH}_{2} \mathrm{Cl}_{2}$. (R)-O-methyl mandeliwc acid $(0.011 \mathrm{~g}, 0.066 \mathrm{mmol})$, DCC $(0.014 \mathrm{~g}, 0.066 \mathrm{mmol})$, and a few crystals of DMAP were added, and the mixture was stirred for $15 \mathrm{~min}$. The solvent was removed under vacuum, and the residue was purified by flash chromatography on silica gel (100\% hexanes), affording the desired ester $(0.008 \mathrm{~g}, 53 \%)$ yield as a clear oil. ${ }^{1} \mathrm{H}$ NMR $\left(\mathrm{CDCl}_{3}\right)$ 87.45-7.30 (m, 5H), $5.50(\mathrm{~d}, \mathrm{~J}=5.1 \mathrm{~Hz}, 1 \mathrm{H}), 4.80(\mathrm{~s}, 1 \mathrm{H}), 3.43(\mathrm{~s}, 3 \mathrm{H}), 3.31$ (A of ABX, J = 6.3 Hz, 9.9 Hz, 1H), 3.12 (B of ABX, J = 6.3 Hz, $9.9 \mathrm{~Hz}, 1 \mathrm{H}), 1.98-1.85$ (m, 1H), 0.82 (d, J $=6.3 \mathrm{~Hz}, 3 \mathrm{H}), 0.81(\mathrm{~s}, 9 \mathrm{H}), 0.15(\mathrm{~s}, 9 \mathrm{H}),-0.09(\mathrm{~s}, 3 \mathrm{H}),-0.12(\mathrm{~s}, 3 \mathrm{H})$.

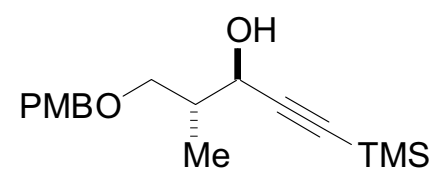

\section{(3R, 4R)-5-(p-Methoxybenzyl)-4-methyl-1-trimethylsilyl-1-pentyn-3-ol (2b)}

The standard procedure for TMS acetylene addition was employed using aldehyde $\mathbf{1 b}(0.090 \mathrm{~g}, 0.44$ mmol), TMS acetylene (0.25 mL, $1.8 \mathrm{mmol}), \mathrm{Et}_{2} \mathrm{Zn}(0.18 \mathrm{~mL}, 1.8 \mathrm{mmol}), \mathrm{Ti}(\mathrm{OiPr})_{4}(0.13 \mathrm{~mL}, 0.44 \mathrm{mmol})$, and $S$-BINOL $(0.052 \mathrm{~g}, 0.18 \mathrm{mmol})$. During the workup, the organic extracts were washed with $10 \%$ $\mathrm{NaOH}$ before the brine wash to remove the BINOL residue, which co-spotted with the product. Flash 
chromatography on silica gel $(2.5 \% \mathrm{EtOAc} /$ hexanes to $10 \%$ EtOAc/ hexanes gradient) afforded alcohol $\mathbf{2 b}$ $(0.090 \mathrm{~g}, 69 \%$ yield, $6: 1$ mixture of diastereomers $)$ as a clear oil. $\mathrm{R}_{\mathrm{f}}=0.05(20 \%$ EtOAc/ hexanes $) ;{ }^{1} \mathrm{H}$ NMR $\left(\mathrm{CDCl}_{3}\right.$, major diastereomer) $\delta 7.24(\mathrm{~d}, \mathrm{~J}=8.7 \mathrm{~Hz}, 2 \mathrm{H}), 6.87(\mathrm{~d}, \mathrm{~J}=8.7 \mathrm{~Hz}, 2 \mathrm{H}), 4.45(\mathrm{~d}, \mathrm{~J}=3.0 \mathrm{~Hz}$, 2H), $4.38(\mathrm{t}, \mathrm{J}=6.3 \mathrm{~Hz}, 1 \mathrm{H}), 2.80(\mathrm{~s}, 3 \mathrm{H}), 3.68(\mathrm{dd}, \mathrm{J}=4.2 \mathrm{~Hz}, 9.3 \mathrm{~Hz}, 1 \mathrm{H}), 3.42(\mathrm{dd}, \mathrm{J}=6.9 \mathrm{~Hz}, 9.3 \mathrm{~Hz}$, $1 \mathrm{H}), 3.13(\mathrm{~d}, \mathrm{~J}=5.4 \mathrm{~Hz}, 1 \mathrm{H}), 2.09-2.02(\mathrm{~m}, 1 \mathrm{H}), 1.03(\mathrm{~d}, \mathrm{~J}=6.6 \mathrm{~Hz}, 3 \mathrm{H}), 0.17(\mathrm{~s}, 9 \mathrm{H})$; Anal. Calcd for $\mathrm{C}_{17} \mathrm{H}_{26} \mathrm{O}_{3} \mathrm{Si}: \mathrm{C}, 66.62, \mathrm{H}, 8.55$, Found: $\mathrm{C}, 66.69, \mathrm{H}, 8.72$.

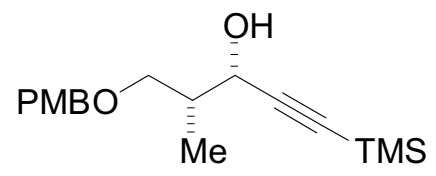

(3S, 4R)-5-(p-Methoxybenzyl)-4-methyl-1-trimethylsilyl-1-pentyn-3-ol (3b)

The standard procedure for TMS acetylene addition was employed using aldehyde 1b $(0.090 \mathrm{~g}, 0.44$ mmol), TMS acetylene (0.25 mL, $1.8 \mathrm{mmol}), \mathrm{Et}_{2} \mathrm{Zn}(0.18 \mathrm{~mL}, 1.8 \mathrm{mmol})$, Ti( $(\mathrm{OiPr})_{4}(0.13 \mathrm{~mL}, 0.44 \mathrm{mmol})$, and $R$-BINOL $(0.052 \mathrm{~g}, 0.18 \mathrm{mmol})$. During the workup, the organic extracts were washed with $10 \%$ $\mathrm{NaOH}$ before the brine wash to remove the BINOL residue, which co-spotted with the product. Flash chromatography on silica gel $(2.5 \% \mathrm{EtOAc} /$ hexanes to $10 \% \mathrm{EtOAc} /$ hexanes gradient) afforded alcohol $\mathbf{3 b}$ ( $0.10 \mathrm{~g}, 77 \%$ yield, $7: 1$ mixture of diastereomers $)$ as a clear oil. $\mathrm{R}_{\mathrm{f}}=0.05\left(20 \%\right.$ EtOAc/ hexanes); ${ }^{1} \mathrm{H}$ NMR $\left(\mathrm{CDCl}_{3}\right.$, major diastereomer) $\delta 7.25(\mathrm{~d}, \mathrm{~J}=8.7 \mathrm{~Hz}, 2 \mathrm{H}), 6.88(\mathrm{~d}, \mathrm{~J}=8.7 \mathrm{~Hz}, 2 \mathrm{H}), 4.45(\mathrm{~d}, \mathrm{~J}=2.7 \mathrm{~Hz}, 2 \mathrm{H})$, 4.42-4.36 (m, 1H), $3.80(\mathrm{~s}, 3 \mathrm{H}), 3.68(\mathrm{t}, \mathrm{J}=9.0 \mathrm{~Hz}, 1 \mathrm{H}), 3.50$ (dd, J = 4.2 Hz, $9.3 \mathrm{~Hz}, 1 \mathrm{H}), 2.30-2.18$ (m, 1h), 0.90 (d, J = 7.2 Hz, 3H), 0.17 (s, 9H); Anal. Calcd for $\mathrm{C}_{17} \mathrm{H}_{26} \mathrm{O}_{3} \mathrm{Si}: \mathrm{C}, 66.62, \mathrm{H}, 8.55$, Found: $\mathrm{C}, 66.48$, H, 8.48 .

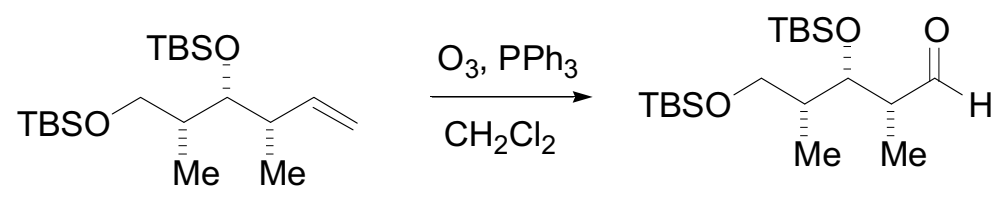

$(2 R, 3 S, 4 R)-3,5-B i s(t$-butyldimethylsilyloxy)-2,4-dimethylpentanal (4)

The above alkene ${ }^{1}(0.30 \mathrm{~g}, 0.81 \mathrm{mmol})$ was taken up in $8 \mathrm{~mL}$ of $\mathrm{CH}_{2} \mathrm{Cl}_{2}$ and chilled to $-78{ }^{\circ} \mathrm{C} . \mathrm{O}_{3}$ was bubbled through the solution until a blue color persisted. Ar was then bubbled through the reaction mixture for $15 \mathrm{~min}$. $\mathrm{PPh}_{3}(0.32 \mathrm{~g}, 1.21 \mathrm{mmol})$ was then added, and the mixture was warmed to room temperature for $3 \mathrm{~h}$. The solvent was removed under reduced pressure and the residue was triturated with $10 \% \mathrm{EtOAc} /$ hexanes and filtered through Celite to remove triphenylphosphine oxide. The solvent was removed under reduced pressure and the residue was purified by flash chromatography on silica gel (1\% EtOAc/ hexanes to $2.5 \% \mathrm{EtOAC} /$ hexanes gradient), affording aldehyde 4 (0.21 g, 70\% yield) as a clear oil. $\mathrm{R}_{\mathrm{f}}=0.20(5 \%$ EtOAc/ hexanes $) ;[\alpha]^{\mathrm{D}}{ }_{20}=-40.9\left(\mathrm{c}=1.45, \mathrm{CHCl}_{3}\right) ; \mathrm{IR}($ film $) 1728 \mathrm{~cm}^{-1} ;{ }^{1} \mathrm{H} \mathrm{NMR}\left(\mathrm{CDCl}_{3}\right) \delta$ $9.87(\mathrm{~d}, \mathrm{~J}=0.6 \mathrm{~Hz}, 1 \mathrm{H}), 4.23(\mathrm{dd}, \mathrm{J}=3.6 \mathrm{~Hz}, 5.4 \mathrm{~Hz}, 1 \mathrm{H}), 3.47$ (A of ABX, J = 7.5 Hz, 10.2 Hz, 1H), 3.40 (B of ABX, J = 5.7 Hz, 10.2 Hz, 1H), 2.64-2.55 (m, 1H), 1.82-1.70 (m, 1H), $1.05(\mathrm{~d}, \mathrm{~J}=7.2 \mathrm{~Hz}, 3 \mathrm{H}), 0.89$ 
(s, 9H), $0.88(\mathrm{~s}, 9 \mathrm{H}), 0.81(\mathrm{~d}, \mathrm{~J}=6.9 \mathrm{~Hz}, 3 \mathrm{H}), 0.09(\mathrm{~s}, 3 \mathrm{H}), 0.06(\mathrm{~s}, 3 \mathrm{H}), 0.03(\mathrm{~s}, 6 \mathrm{H}) ;{ }^{13} \mathrm{C} \mathrm{NMR}\left(\mathrm{CDCl}_{3}\right) \delta$ 205.41, 72.06, 65.25, 51.60, 39.13, 25.92, 25.85, 18.23, 18.16, 11.54, 9.44, -4.11, -4.54, -5.40.

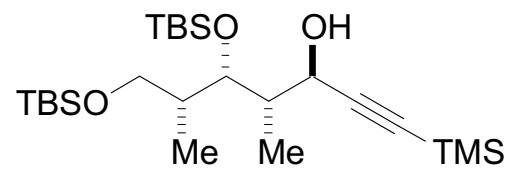

(3R, 4R, 5S, 6R)-5,7-Bis(t-butyldimethylsilyloxy)-4,6-dimethyl-1-trimethylsilyl-1-heptyn-3-ol (5)

The standard procedure for TMS acetylene addition was employed using aldehyde 4 ( $0.10 \mathrm{~g}, 0.27 \mathrm{mmol})$, TMS acetylene $(0.15 \mathrm{~mL}, 1.1 \mathrm{mmol}), \mathrm{Et}_{2} \mathrm{Zn}(0.11 \mathrm{~mL}, 1.1 \mathrm{mmol})$, Ti(OiPr $)_{4}(0.080 \mathrm{~mL}, 0.27 \mathrm{mmol})$, and $(S)$-BINOL (0.031 g, $0.11 \mathrm{mmol})$, affording alcohol 5 (0.12 g, 92\% yield, 1.6:1 mixture of diastereomers) as a clear oil. $\mathrm{R}_{\mathrm{f}}=0.1\left(5 \%\right.$ EtOAc/ hexanes); ${ }^{1} \mathrm{H} \mathrm{NMR}\left(\mathrm{CDCl}_{3}\right.$, major diastereomer $) \delta 4.34(\mathrm{dd}, \mathrm{J}=3.5 \mathrm{~Hz}$, $8.5 \mathrm{~Hz}, 1 \mathrm{H}), 4.04(\mathrm{t}, \mathrm{J}=3.0 \mathrm{~Hz}, 1 \mathrm{H}), 3.43(\mathrm{~A}$ of ABX, J = 7.5 Hz, $10.0 \mathrm{~Hz}, 1 \mathrm{H}), 3.38$ (B of ABX, J = 7.0 $\mathrm{Hz}, 9.5 \mathrm{~Hz}, 1 \mathrm{H}), 3.17(\mathrm{~d}, \mathrm{~J}=3.5 \mathrm{~Hz}, 1 \mathrm{H}), 2.00-1.80(\mathrm{~m}, 2 \mathrm{H}), 0.97(\mathrm{~d}, \mathrm{~J}=7.0 \mathrm{~Hz}, 3 \mathrm{H}), 0.92-0.88(\mathrm{~m}, 21 \mathrm{H})$, $0.17(\mathrm{~s}, 9 \mathrm{H}), 0.12(\mathrm{~s}, 3 \mathrm{H}), 0.08(\mathrm{~s}, 3 \mathrm{H}), 0.04(\mathrm{~s}, 6 \mathrm{H})$; Anal. Calcd for $\mathrm{C}_{24} \mathrm{H}_{52} \mathrm{O}_{3} \mathrm{Si}_{3}: \mathrm{C}, 60.95, \mathrm{H}, 11.08$, Found: C, 61.18, H, 11.28.

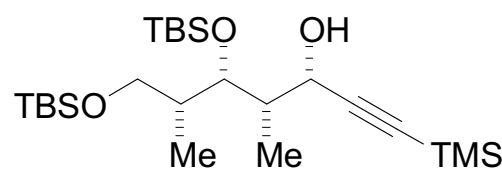

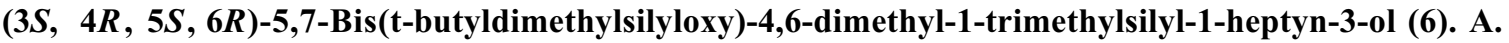
With (R)-BINOL as the Chiral Ligand.

The standard procedure for TMS acetylene addition was employed using aldehyde 4 ( $0.10 \mathrm{~g}, 0.27$ mmol), TMS acetylene $(0.15 \mathrm{~mL}, 1.1 \mathrm{mmol}), \mathrm{Et}_{2} \mathrm{Zn}(0.11 \mathrm{~mL}, 1.1 \mathrm{mmol}), \mathrm{Ti}(\mathrm{OiPr})_{4}(0.080 \mathrm{~mL}, 0.27$ $\mathrm{mmol})$, and $(R)$-BINOL $(0.031 \mathrm{~g}, 0.11 \mathrm{mmol})$, affording alcohol $6(0.11 \mathrm{~g}, 85 \%$ yield, 7:1 mixture of diastereomers) as a clear oil. $\mathrm{R}_{\mathrm{f}}=0.1(5 \%$ EtOAc/ hexanes $) ;[\alpha]_{20}^{\mathrm{D}_{20}}=-1.7\left(\mathrm{c}=1.30, \mathrm{CHCl}_{3}\right) ; \mathrm{IR}(\mathrm{film})$ 3450, $2174 \mathrm{~cm}^{-1} ;{ }^{1} \mathrm{H} \mathrm{NMR}\left(\mathrm{CDCl}_{3}\right.$, major diastereomer) $\delta 4.31(\mathrm{~d}, \mathrm{~J}=7.0 \mathrm{~Hz}, 1 \mathrm{H}), 3.94(\mathrm{t}, \mathrm{J}=4.0 \mathrm{~Hz}, 1 \mathrm{H})$, 3.55 (A of ABX, J = 6.5 Hz, 10.0 Hz, 1H), 3.40 (B of ABX, J = 7.0 Hz, 9.5 Hz, 1H), 2.09 (broad s, 1H), 1.92-1.82 (m, 2H), 1.02 (d, J = 7.0 Hz, 3H), 0.92 (d, J = 7.0 Hz, 3H), 0.90 (s, 9H), 0.90 (s, 9H), 0.17 (s, 9H), 0.09 (s, 3H), 0.07 (s, 3H), 0.04 (s, 6H); ${ }^{13} \mathrm{C} \mathrm{NMR}\left(\mathrm{CDCl}_{3}\right) \delta 106.51,90.44,73.91,66.02,65.67,43.25$, $40.50,26.10,25.93,18.41,18.25,12.73,10.78,-0.15,-4.00,-4.06$, -5.37; Anal. Calcd for $\mathrm{C}_{24} \mathrm{H}_{52} \mathrm{O}_{3} \mathrm{Si}_{3}: \mathrm{C}$, 60.95, H, 11.08, Found: C, 61.20, H, 11.18.

\section{B. Without BINOL}

The standard procedure for TMS acetylene addition was employed using aldehyde 4 ( $0.064 \mathrm{~g}, 0.17$ mmol), TMS acetylene $(0.14 \mathrm{~mL}, 1.0 \mathrm{mmol}), \mathrm{Et}_{2} \mathrm{Zn}(0.10 \mathrm{~mL}, 1.0 \mathrm{mmol})$, and $\mathrm{Ti}(\mathrm{OiPr})_{4}(0.050 \mathrm{~mL}, 0.17$ mmol), affording alcohol 6 ( $0.10 \mathrm{~g}, 77 \%$ yield, 2:1 mixture) as a clear oil. All analytical data matched the previously characterized material. 


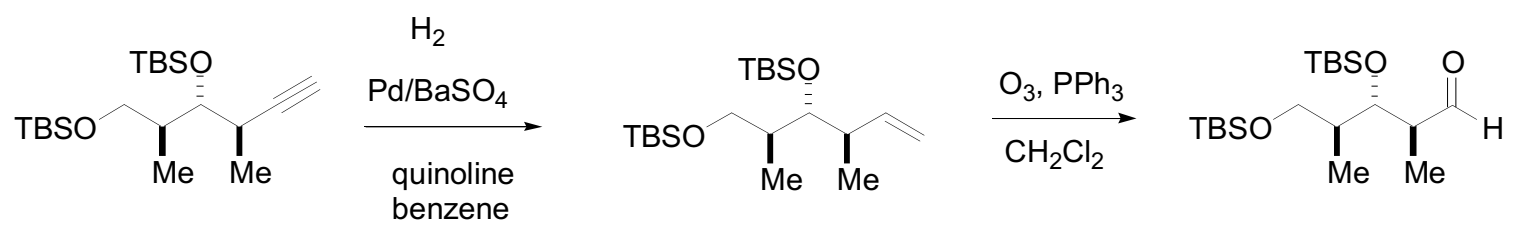

(2S, 3S, 4S)-3,5-Bis(t-butyldimethylsilyloxy)-2,4-dimethylpentanal (7)

The above alkyne ${ }^{2}(0.32 \mathrm{~g}, 0.86 \mathrm{mmol})$ was taken up in $10 \mathrm{~mL}$ of benzene. $5 \% \mathrm{Pd} / \mathrm{BaSO}_{4}$ (reduced, $0.32 \mathrm{~g}$ ) and quinoline $\left(0.10 \mathrm{~mL}\right.$ ) were added, and the mixture was stirred for $5 \mathrm{~min}$. $\mathrm{H}_{2}$ was bubbled through the mixture for $1 \mathrm{~h}$. The mixture was then filtered through Celite and the solvent was removed under reduced pressure. The crude residue was purified by flash chromatography on silica gel ( $100 \%$ hexanes), affording the corresponding alkene $(0.31 \mathrm{~g}, 97 \%$ yield $)$ as a yellow oil. $\mathrm{R}_{\mathrm{f}}=0.50(2.5 \%$ EtOAc/ hexanes); IR (film) $1641 \mathrm{~cm}^{-1} ;{ }^{1} \mathrm{H}$ NMR $\left(\mathrm{CDCl}_{3}\right) \delta$ 5.95-5.87 (m, $\left.1 \mathrm{H}\right), 5.00-4.94(\mathrm{~m}, 2 \mathrm{H}), 3.67$ (A of ABX, J = 5.0 Hz, 10.0 Hz, 1H), $3.53(\mathrm{dd}, \mathrm{J}=3.5 \mathrm{~Hz}, 6.5 \mathrm{~Hz}, 1 \mathrm{H}), 3.42(\mathrm{~B}$ of ABX, J = 7.5 Hz, $10.0 \mathrm{~Hz}$, 1H), 2.42-2.36 (m, 1H), 1.82-1.76 (m, 1H), $1.03(\mathrm{~d}, \mathrm{~J}=7.0 \mathrm{~Hz}, 3 \mathrm{H}), 0.91(\mathrm{~s}, 9 \mathrm{H}), 0.90$ (s, 9H), 0.87 (d, J = $7.0 \mathrm{~Hz}, 3 \mathrm{H}), 0.05(\mathrm{~s}, 6 \mathrm{H}), 0.04(\mathrm{~s}, 6 \mathrm{H}) ;{ }^{13} \mathrm{C} \mathrm{NMR}\left(\mathrm{CDCl}_{3}\right) \delta 141.54,113.69,77.44,65.42,41.66,40.47$, 26.12, 25.96, 18.38, 18.30, 14.12, -3.99, -5.28, -5.39; Anal. Calcd for $\mathrm{C}_{20} \mathrm{H}_{44} \mathrm{O}_{2} \mathrm{Si}_{2}: \mathrm{C}, 64.45, \mathrm{H}, 11.90$, Found: C, 64.71, H, 11.91 .

The aforementioned alkene $(0.23 \mathrm{~g}, 0.62 \mathrm{mmol})$ was taken up in $10 \mathrm{~mL}$ of $\mathrm{CH}_{2} \mathrm{Cl}_{2}$ and chilled to $-78{ }^{\circ} \mathrm{C} . \mathrm{O}_{3}$ was bubbled through the solution until a blue color persisted. Ar was then bubbled through the reaction mixture for $15 \mathrm{~min}$. $\mathrm{PPh}_{3}(0.24 \mathrm{~g}, 0.93 \mathrm{mmol})$ was then added, and the mixture was warmed to room temperature for $3 \mathrm{~h}$. The solvent was removed under reduced pressure and the residue was triturated with $10 \%$ EtOAc/ hexanes and filtered through Celite to remove triphenylphosphine oxide. The solvent was removed under reduced pressure and the residue was purified by flash chromatography on silica gel (1\% EtOAc/ hexanes to $2.5 \% \mathrm{EtOAC} /$ hexanes gradient), affording aldehyde $7(0.20 \mathrm{~g}, 87 \%$ yield $)$ as a clear oil. $\mathrm{R}_{\mathrm{f}}=0.20\left(5 \%\right.$ EtOAc/ hexanes); $[\alpha]_{20}^{\mathrm{D}}=+26.1\left(\mathrm{c}=1.38, \mathrm{CHCl}_{3}\right) ; \mathrm{IR}$ (film) $1728 \mathrm{~cm}^{-1} ;{ }^{1} \mathrm{H}$ NMR $\left(\mathrm{CDCl}_{3}\right) \delta 9.79(\mathrm{~d}, \mathrm{~J}=3.0 \mathrm{~Hz}, 1 \mathrm{H}), 4.01(\mathrm{dd}, \mathrm{J}=3.0 \mathrm{~Hz}, 5.5 \mathrm{~Hz}, 1 \mathrm{H}), 3.58(\mathrm{~A}$ of ABX, J = 6.0 Hz, $10.5 \mathrm{~Hz}$, 1H), 3.51 (B of ABX, J = 6.0 Hz, 9.5 Hz, 1H), 2.60-2.54 (m, 1H), 1.98-1.90 (m, 1H), 1.13 (d, J = 7.0 Hz, $3 \mathrm{H}), 0.89(\mathrm{~s}, 18 \mathrm{H}), 0.86(\mathrm{~d}, \mathrm{~J}=7.5 \mathrm{~Hz}, 3 \mathrm{H}), 0.08(\mathrm{~s}, 3 \mathrm{H}), 0.07$ (s, 3H), $0.04(\mathrm{~s}, 3 \mathrm{H}), 0.04(\mathrm{~s}, 3 \mathrm{H}) ;{ }^{13} \mathrm{C} \mathrm{NMR}$ $\left(\mathrm{CDCl}_{3}\right) \delta 205.07,75.38,64.89,49.30,41.03,25.87,18.14,12.81,11.99,-4.33,-4.56,-5.41,-5.55$.

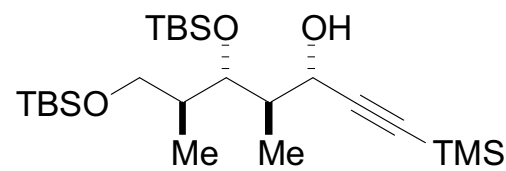

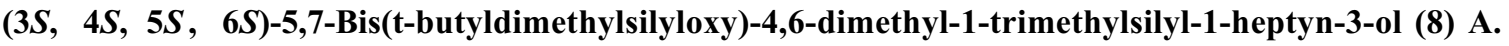
From TMS Acetylene Addition to Aldehyde 7 with (S)-Binol as the Chiral Ligand.

The standard procedure for TMS acetylene addition was employed using aldehyde 7 ( $0.075 \mathrm{~g}, 0.20$ mmol), TMS acetylene $(0.11 \mathrm{~mL}, 0.80 \mathrm{mmol}), \mathrm{Et}_{2} \mathrm{Zn}(0.082 \mathrm{~mL}, 0.080 \mathrm{mmol}), \mathrm{Ti}(\mathrm{OiPr})_{4}(0.059 \mathrm{~mL}, 0.20$ mmol), and ( $R$ )-BINOL (0.023 g, $0.080 \mathrm{mmol})$, affording alcohol $8(0.055 \mathrm{~g}, 59 \%$ yield, 6:1 mixture of diastereomers $)$ as a clear oil. $\mathrm{R}_{\mathrm{f}}=0.1(5 \%$ EtOAc/ hexanes $) ;{ }^{1} \mathrm{H}$ NMR $\left(\mathrm{CDCl}_{3}\right.$, major diastereomer $) \delta 4.44$ 
$(\mathrm{d}, \mathrm{J}=7.5 \mathrm{~Hz}, 1 \mathrm{H}), 3.78(\mathrm{dd}, \mathrm{J}=3.6 \mathrm{~Hz}, 6.6 \mathrm{~Hz}, 1 \mathrm{H}), 3.60$ (A of ABX, J = 7.5 Hz, 10.2 Hz, 1H), 3.43 (B of ABX, J = 6.6 Hz, 10.2 Hz, 1H), $3.09(\mathrm{~s}, 1 \mathrm{H}), 2.04-1.96(\mathrm{~m}, 2 \mathrm{H}), 1.01(\mathrm{~d}, \mathrm{~J}=6.9 \mathrm{~Hz}, 3 \mathrm{H}), 0.92(\mathrm{~d}, \mathrm{~J}=6.9$ Hz, 3H), 0.91 (s, 9H), 0.89 (s, 9H), 0.17 (s, 9H), 0.12 (s, 3H), 0.10 (s, 3H), 0.04 (s, 6H).

\section{B. Without BINOL}

The standard procedure for TMS acetylene addition was employed using aldehyde 7 (0.10 g, 0.21

mmol), TMS acetylene (0.18 mL, $1.3 \mathrm{mmol}), \mathrm{Et}_{2} \mathrm{Zn}(0.13 \mathrm{~mL}, 1.3 \mathrm{mmol})$, and Ti(OiPr) ${ }_{4}(0.062 \mathrm{~mL}, 0.21$ mmol), affording alcohol 8 (0.10 g, 77\% yield, 1.3:1 mixture in favor the 3(S) diastereomer) as a clear oil. All analytical data matched the previously characterized material.

\section{From Noyori reduction of ketone 13}

Ketone 13 (0.052 g, $0.11 \mathrm{mmol})$ was taken up in $1.5 \mathrm{~mL}$ iPrOH. Catalyst ent-14 (0.0032 g, 0.0055 mmol) was added, and the mixture was stirred overnight. The solvent was removed under reduced pressure, and the residue was purified by flash chromatography on silica gel (1\% EtOAc/ hexanes to $2.5 \%$ EtOAc/ hexanes gradient), affording alcohol $8(0.038 \mathrm{~g}, 73 \%$ yield $)$ as a light yellow oil. $\mathrm{R}_{\mathrm{f}}=0.2(5 \%$ EtOAc/ hexanes). The NMR spectra of this material were identical to those of the material prepared in part A.

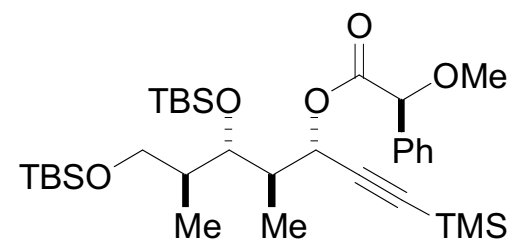

\section{(S)-O-Methyl Mandelic Ester of 8}

Alcohol 8 (0.010 g, $0.021 \mathrm{mmol})$ was taken up in $1 \mathrm{~mL}$ of $\mathrm{CH}_{2} \mathrm{Cl}_{2}$. (S)-O-Methyl mandelic acid $(0.0070 \mathrm{~g}, 0.042 \mathrm{mmol})$, DCC $(0.0087 \mathrm{~g}, 0.042 \mathrm{mmol})$, and a few crystals of DMAP were added. The mixture was stirred for $15 \mathrm{~min}$, and the solvent was removed under vacuum. The residue was purified by flash chromatography on silica gel (100\% hexanes), affording the desired ester $(0.008 \mathrm{~g}, 62 \%)$ yield as a clear oil. ${ }^{1} \mathrm{H}$ NMR $\left(\mathrm{CDCl}_{3}\right) \delta$ 7.46-7.31 (m, 5H), $5.67(\mathrm{~d}, \mathrm{~J}=4.8 \mathrm{~Hz}, 1 \mathrm{H}), 4.76(\mathrm{~s}, 1 \mathrm{H}), 3.70-3.58(\mathrm{~m}, 2 \mathrm{H})$, 3.44 (s, 3H), 3.36 (B of ABX, J = 7.2 Hz, 9.9 Hz, 1H), 2.16-1.88 (m, 2H), 0.98 (d, J = 6.9 Hz, 3H), 0.92$0.86(\mathrm{~m}, 21 \mathrm{H}), 0.14(\mathrm{~s}, 3 \mathrm{H}), 0.14(\mathrm{~s}, 3 \mathrm{H}) 0.08(\mathrm{~s}, 9 \mathrm{H}), 0.02(\mathrm{~s}, 3 \mathrm{H}), 0.02(\mathrm{~s}, 3 \mathrm{H})$.

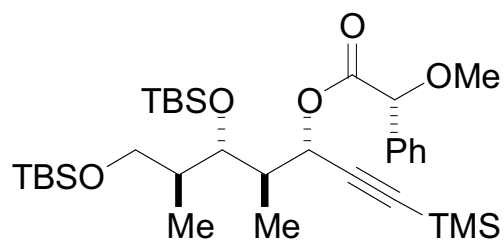

\section{(R)-O-Methyl Mandelic Ester of 8}

Alcohol 8 (0.010 g, $0.021 \mathrm{mmol})$ was taken up in $1 \mathrm{~mL}$ of $\mathrm{CH}_{2} \mathrm{Cl}_{2}$. (S)-O-Methyl mandelic acid (0.0070 g, $0.042 \mathrm{mmol})$, DCC $(0.0087 \mathrm{~g}, 0.042 \mathrm{mmol})$, and a few crystals of DMAP were added. The mixture was stirred for $15 \mathrm{~min}$, and the solvent was removed under vacuum. The residue was purified by 
flash chromatography on silica gel (100\% hexanes), affording the desired ester $(0.009 \mathrm{~g}, 69 \%)$ yield as a clear oil. ${ }^{1} \mathrm{H}$ NMR $\left(\mathrm{CDCl}_{3}\right) \delta$ 7.45-, $7.30(\mathrm{~m}, 5 \mathrm{H}), 5.70(\mathrm{~d}, \mathrm{~J}=5.1 \mathrm{~Hz}, 1 \mathrm{H}), 4.78(\mathrm{~s}, 1 \mathrm{H}), 3.56(\mathrm{~A}$ of ABX, J $=6.6 \mathrm{~Hz}, 10.2 \mathrm{~Hz}, 1 \mathrm{H}), 3.50(\mathrm{dd}, \mathrm{J}=2.7 \mathrm{~Hz}, 8.4 \mathrm{~Hz}, 1 \mathrm{H}), 3.42(\mathrm{~s}, 9 \mathrm{H}), 3.26(\mathrm{~B}$ of ABX, J = 7.2 Hz, 9.9 Hz, 1H), 1.94-1.70 (m, 2H), 0.89-0.85 (m, 21H), $0.80(\mathrm{~d}, \mathrm{~J}=7.2 \mathrm{~Hz}, 3 \mathrm{H}), 0.15(\mathrm{~s}, 9 \mathrm{H}), 0.12(\mathrm{~s}, 3 \mathrm{H}), 0.03$ (s, $3 \mathrm{H}),-0.01$ (s, 3H), -0.03 (s, 3H).

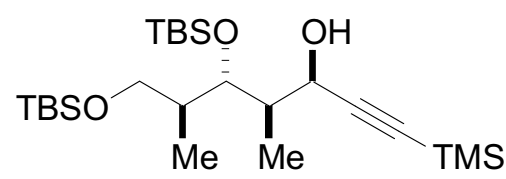

(3R, $4 S, 5 S, 6 S)$-5,7-Bis(t-butyldimethylsilyloxy)-4,6-dimethyl-1-trimethylsilyl-1-heptyn-3-ol (9) A. From TMS Acetylene Addition to Aldehyde 7.

The standard procedure for TMS acetylene addition was employed using aldehyde 7 (0.075g, 0.20 $\mathrm{mmol})$, TMS acetylene $(0.11 \mathrm{~mL}, 0.80 \mathrm{mmol}), \mathrm{Et}_{2} \mathrm{Zn}(0.082 \mathrm{~mL}, 0.080 \mathrm{mmol}), \mathrm{Ti}(\mathrm{OiPr})_{4}(0.059 \mathrm{~mL}, 0.20$ mmol), and (S)-BINOL (0.023 g, $0.080 \mathrm{mmol})$, affording alcohol 9 (0.063 g, 67\% yield, 6:1 mixture of diastereomers $)$ as a clear oil. $\mathrm{R}_{\mathrm{f}}=0.2(5 \% \mathrm{EtOAc} /$ hexanes $) ;[\alpha]_{20}^{\mathrm{D}}=+9.9\left(\mathrm{c}=1.62, \mathrm{CHCl}_{3}\right) ; \mathrm{IR}(\mathrm{film})$ 3441, $2182 \mathrm{~cm}^{-1} ;{ }^{1} \mathrm{H}$ NMR $\left(\mathrm{CDCl}_{3}\right) \delta 4.73(3.0 \mathrm{~Hz}, 1 \mathrm{H}), 3.90(\mathrm{t}, \mathrm{J}=5.0 \mathrm{~Hz}, 1 \mathrm{H}), 3.61$ (A of ABX, J = 3.5 $\mathrm{Hz}, 10 \mathrm{~Hz}, 1 \mathrm{H}), 3.50-3.47(\mathrm{~m}, 2 \mathrm{H}), 2.00-1.95(\mathrm{~m}, 2 \mathrm{H}), 1.13(\mathrm{~d}, \mathrm{~J}=7.5 \mathrm{~Hz}, 3 \mathrm{H}), 0.94(\mathrm{~d}, \mathrm{~J}=7.0 \mathrm{~Hz}, 3 \mathrm{H})$, 0.91 (s, 9H), 0.89 (s, 9H), 0.16 (s, 9H), 0.14 (s, 3H), 0.11 (s, 3H), 0.04 (s, 6H); ${ }^{13} \mathrm{C} \mathrm{NMR}\left(\mathrm{CDCl}_{3}\right) \delta 106.03$, $89.19,77.78,64.92,64.72,40.92,39.85,26.07,25.89,18.24,18.20,13.14,13.12,-0.08,-4.09,-4.25,-5.40$, -5.50; Anal. Calcd for $\mathrm{C}_{24} \mathrm{H}_{52} \mathrm{O}_{3} \mathrm{Si}_{3}$ : C, 60.95, H, 11.08, Found: C, 60.91, H, 11.23.

\section{B: From Noyori Reduction of Ketone 13}

Ketone 13 (0.054 g, $0.11 \mathrm{mmol})$ was taken up in $1.5 \mathrm{~mL}$ of iPrOH. Catalyst $14(0.0034 \mathrm{~g}, 0.0057$ mmol) was added, and the mixture was stirred overnight. The solvent was removed under reduced pressure, and the residue was purified by flash chromatography on silica gel (1\% EtOAc/ hexanes to $2.5 \%$ EtOAc/ hexanes), affording alcohol $9(0.041 \mathrm{~g}, 76 \%$ yield $)$ as a light yellow oil. $\mathrm{R}_{\mathrm{f}}=0.2(5 \% \mathrm{EtOAc} /$ hexanes); The NMR spectra of this material were superimposable with those of material prepared in part A.

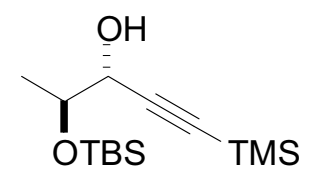

(3R, 4S)-4-(t-Butyldimethylsilyloxy)-1-trimethylsilyl-1-pentyn-3-ol (11). A With (R)-BINOL as the Chiral Ligand.

The standard procedure for TMS acetylene addition was employed using aldehyde $\mathbf{1 0}(0.10 \mathrm{~g}, 0.53$ mmol), TMS acetylene (0.30 mL, $2.1 \mathrm{mmol}), \mathrm{Et}_{2} \mathrm{Zn}(0.22 \mathrm{~mL}, 2.1 \mathrm{mmol})$, Ti(OiPr) $)_{4}(0.16 \mathrm{~mL}, 0.53 \mathrm{mmol})$, and $(R)$-BINOL $(0.061 \mathrm{~g}, 0.21 \mathrm{mmol})$, affording alcohol $11(0.090 \mathrm{~g}, 60 \%$ yield, 11:1 mixture of diastereomers) as a clear oil. $\mathrm{R}_{\mathrm{f}}=0.5(20 \%$ EtOAc/ hexanes $) ;[\alpha]_{20}^{\mathrm{D}}=+2.1\left(\mathrm{c}=1.25, \mathrm{CHCl}_{3}\right) ; \mathrm{IR}$ (film) 3214, $2174 \mathrm{~cm}^{-1} ;{ }^{1} \mathrm{H} \mathrm{NMR}\left(\mathrm{CDCl}_{3}\right.$, major diastereomer) $\delta 4.24(\mathrm{~d}, \mathrm{~J}=3.0 \mathrm{~Hz}, 1 \mathrm{H}), 3.92-3.89(\mathrm{~m}, 1 \mathrm{H}), 2.33$ 
(broad s, 1H), $1.22(\mathrm{dd}, \mathrm{J}=1.0 \mathrm{~Hz}, 6.0 \mathrm{~Hz}, 3 \mathrm{H}), 0.90(\mathrm{~s}, 9 \mathrm{H}), 0.17(\mathrm{~s}, 9 \mathrm{H}), 0.10(\mathrm{~s}, 3 \mathrm{H}), 0.10(\mathrm{~s}, 3 \mathrm{H}) ;{ }^{13} \mathrm{C}$ $\operatorname{NMR}\left(\mathrm{CDCl}_{3}\right) \delta 103.65,90.70,70.99,67.42,25.73,18.17,18.00,-0.20,-4.45,-4.81$.

\section{B. Without BINOL.}

The standard procedure for TMS acetylene addition was employed using aldehyde $10(0.10 \mathrm{~g}, 0.49$ mmol), TMS acetylene $(0.28 \mathrm{~mL}, 2.0 \mathrm{mmol}), \mathrm{Et}_{2} \mathrm{Zn}(0.20 \mathrm{~mL}, 2.0 \mathrm{mmol})$, and $\mathrm{Ti}(\mathrm{OiPr})_{4}(0.15 \mathrm{~mL}, 0.49$ mmol), affording alcohol $11(0.10 \mathrm{~g}, 66 \%$ yield, 6:1 mixture of diastereomers favoring the 3(R) diastereomer) as a clear oil. All analytical data was consistent with the BINOL directed cases.

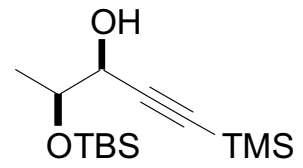

\section{(3S, 4S)-4-(t-Butyldimethylsilyloxy)-1-trimethylsilyl-1-pentyn-3-ol (12)}

The standard procedure for TMS acetylene addition was employed using aldehyde $10(0.10 \mathrm{~g}, 0.53$ mmol), TMS acetylene (0.30 mL, $2.1 \mathrm{mmol}), \mathrm{Et}_{2} \mathrm{Zn}(0.22 \mathrm{~mL}, 2.1 \mathrm{mmol}), \mathrm{Ti}(\mathrm{OiPr})_{4}(0.16 \mathrm{~mL}, 0.53 \mathrm{mmol})$, and $(S)$-BINOL (0.061 g, $0.21 \mathrm{mmol})$, affording alcohol 12 (0.083 g, 55\% yield, 1.3:1 mixture of diastereomers) as a clear oil. $\mathrm{R}_{\mathrm{f}}=0.5$ (20\% EtOAc/ hexanes); ${ }^{1} \mathrm{H} \mathrm{NMR}\left(\mathrm{CDCl}_{3}\right.$, major diastereomer) $\delta$ $4.06(\mathrm{~d}, \mathrm{~J}=5.0 \mathrm{~Hz}, 1 \mathrm{H}), 3.89-3.95(\mathrm{~m}, 1 \mathrm{H}), 1.12$ (d, J = 6.5 Hz, 3H), 0.91 (s, 9H), 0.17 (s, $9 \mathrm{H}), 0.13$ (s, $3 \mathrm{H}), 0.11(\mathrm{~s}, 3 \mathrm{H})$.

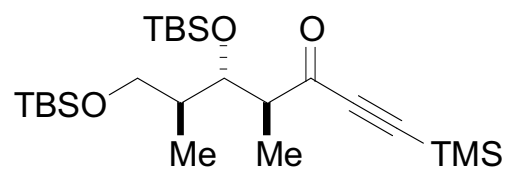

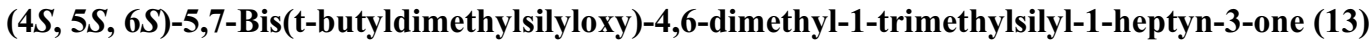

To a solution of alcohol $8(0.085 \mathrm{~g}, 0.18 \mathrm{mmol})$ in $2 \mathrm{~mL}$ of $\mathrm{CH}_{2} \mathrm{Cl}_{2}$ was added $\mathrm{NaHCO}_{3}(0.16 \mathrm{~g}$, $1.8 \mathrm{mmol})$ and Dess-Martin periodinane $(0.11 \mathrm{~g}, 0.27 \mathrm{mmol})$. The heterogeneous mixture was stirred for 30 min and quenched with equal volumes of aq. $\mathrm{NaHCO}_{3}$ and aq. $\mathrm{Na}_{2} \mathrm{~S}_{2} \mathrm{O}_{3}$. After 30 min, the biphasic mixture was partitioned in a separatory funnel and the aqueous portion was extracted three times with $\mathrm{CH}_{2} \mathrm{Cl}_{2}$. The combined organic extracts were dried over $\mathrm{MgSO}_{4}$ and filtered. Concentration under reduced pressure, followed by flash chromatography on silica gel (1\% EtOAc/ hexanes) afforded ketone 13 (0.058 $\mathrm{g}, 68 \%$ yield) as a light yellow oil. $\mathrm{R}_{\mathrm{f}}=0.2(5 \%$ EtOAc/ hexanes $) ;[\alpha]_{20}^{\mathrm{D}}=+37.4\left(\mathrm{c}=1.38, \mathrm{CHCl}_{3}\right) ; \mathrm{IR}$ (film) $1684 \mathrm{~cm}^{-1} ;{ }^{1} \mathrm{H} \mathrm{NMR}\left(\mathrm{CDCl}_{3}\right) \delta 4.29(\mathrm{t}, \mathrm{J}=5.5 \mathrm{~Hz}, 1 \mathrm{H}), 3.65$ (A of ABX, J = 4.5 Hz, 9.5 Hz, 1H), $3.51(\mathrm{~B}$ of $\mathrm{ABX}, \mathrm{J}=6.5 \mathrm{~Hz}, 9.5 \mathrm{~Hz}, 1 \mathrm{H}), 2.88-2.82(\mathrm{~m}, 1 \mathrm{H}), 1.86-1.79(\mathrm{~m}, 1 \mathrm{H}), 1.14(\mathrm{~d}, \mathrm{~J}=7.0 \mathrm{~Hz}, 3 \mathrm{H})$, $0.90(\mathrm{~s}, 9 \mathrm{H}), 0.89(\mathrm{~s}, 9 \mathrm{H}), 0.81(\mathrm{~d}, \mathrm{~J}=7.0 \mathrm{~Hz}, 3 \mathrm{H}), 0.25(\mathrm{~s}, 9 \mathrm{H}), 0.10(\mathrm{~s}, 3 \mathrm{H}), 0.10(\mathrm{~s}, 3 \mathrm{H}), 0.04(\mathrm{~s}, 6 \mathrm{H}) ;{ }^{13} \mathrm{C}$ NMR $\left(\mathrm{CDCl}_{3}\right) \delta 189.39,101.99,98.65,74.41,64.80,54.37,39.36,25.97,25.93,18.29,18.17,13.78$, $10.14,-0.77,-4.50,-5.36,-5.47$. 


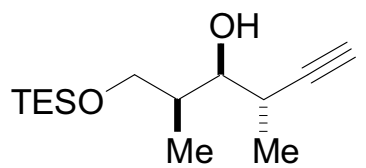

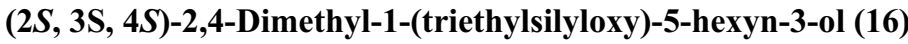

$\mathrm{Pd}(\mathrm{OAc})_{2}(0.13 \mathrm{~g}, 0.59 \mathrm{mmol})$ was taken up in $120 \mathrm{~mL}$ of THF and chilled to -78

${ }^{\circ} \mathrm{C}$. $\mathrm{PPh}_{3}(0.15 \mathrm{~g}, 0.59 \mathrm{mmol})$ was added and the mixture was stirred for $5 \mathrm{~min}$. Aldehyde $\mathbf{1 5}(2.4 \mathrm{~g}, 12$ $\mathrm{mmol})$ and mesylate $16(2.3 \mathrm{~g}, 15 \mathrm{mmol})$ were added in $5 \mathrm{~mL}$ of THF. After $5 \mathrm{~min}, \mathrm{Et}_{2} \mathrm{Zn}(35 \mathrm{~mL}, 1.0 \mathrm{M}$ in THF) was added slowly. The mixture was stirred for five minutes, then warmed to $-20{ }^{\circ} \mathrm{C}$ and stirred overnight. Within five minutes of being warmed to $-20^{\circ} \mathrm{C}$, the reaction mixture turned a dark brown color. The reaction was quenched by slow addition of aq. $\mathrm{NH}_{4} \mathrm{Cl}$ and partitioned in a separatory funnel. The aqueous portion was extracted three times with $\mathrm{Et}_{2} \mathrm{O}$ and the combined organic extracts were washed with brine and dried over $\mathrm{MgSO}_{4}$ with activated charcoal. Filtration through Celite and concentration under reduced pressure, followed by flash chromatography on silica gel (5\% EtOAc/ hexanes) afforded stereotriad $17(2.3 \mathrm{~g}, 76 \%$ yield $)$ as a yellow oil. $\mathrm{R}_{\mathrm{f}}=0.10(5 \% \mathrm{EtOAc} /$ hexanes $) ;[\alpha]_{20}^{\mathrm{D}}=-4.8(\mathrm{c}=1.66$, $\mathrm{CHCl}_{3}$ ); IR (film) $3475 \mathrm{~cm}^{-1} ;{ }^{1} \mathrm{H} \mathrm{NMR}\left(\mathrm{CDCl}_{3}\right) \delta 3.68(\mathrm{~d}, \mathrm{~J}=5.0 \mathrm{~Hz}, 2 \mathrm{H}), 3.62(\mathrm{dd}, \mathrm{J}=3.5 \mathrm{~Hz}, 7.0 \mathrm{~Hz}$, $1 \mathrm{H}), 2.77(\mathrm{~s}, 1 \mathrm{H}), 2.68-2.62(\mathrm{~m}, 1 \mathrm{H}), 2.13(\mathrm{~d}, \mathrm{~J}=2.0 \mathrm{~Hz}, 1 \mathrm{H}), 1.84-1.78(\mathrm{~m}, 1 \mathrm{H}), 1.19(\mathrm{~d}, \mathrm{~J}=7.0 \mathrm{~Hz}, 3 \mathrm{H})$, $0.96(\mathrm{t}, \mathrm{J}=8.5 \mathrm{~Hz}, 9 \mathrm{H}), 0.96(\mathrm{~d}, \mathrm{~J}=7.0 \mathrm{~Hz}, 3 \mathrm{H}), 0.60(\mathrm{q}, \mathrm{J}=8.0 \mathrm{~Hz}, 6 \mathrm{H}) ;{ }^{13} \mathrm{C} \mathrm{NMR}\left(\mathrm{CDCl}_{3}\right) \delta 86.30$, 75.86, 70.02, 66.75, 37.32, 30.43, 17.40, 10.09, 6.57, 4.12; Anal. Calcd for $\mathrm{C}_{14} \mathrm{H}_{28} \mathrm{O}_{2} \mathrm{Si}$ : C, 65.57, H, 11.00, Found: C, 65.39, H, 11.02.

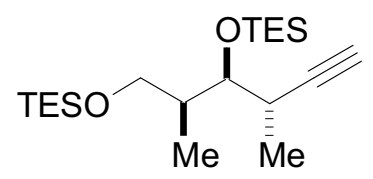

\section{$(3 S, 4 S, 5 S)-3,5-D i m e t h y l-4,6-($ triethylsilyloxy)-1-hexyne (18)}

Alcohol 17 (2.7 g, $11 \mathrm{mmol})$ was taken up in $110 \mathrm{~mL}$ of $\mathrm{CH}_{2} \mathrm{Cl}_{2}$ and chilled to $0{ }^{\circ} \mathrm{C}$. 2,6-lutidine $(2.5 \mathrm{~mL}, 21 \mathrm{mmol})$ and TESOTf $(3.6 \mathrm{~mL}, 16 \mathrm{mmol})$ were added sequentially, and the mixture was stirred

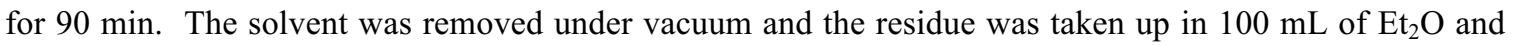
washed successively with aq $\mathrm{NH}_{4} \mathrm{Cl}$, aq. $\mathrm{CuSO}_{4}$, and brine. The ethereal solution was dried over $\mathrm{MgSO}_{4}$ and filtered. Concentration under reduced pressure, followed by flash chromatography on silica gel (100\% hexanes) afforded bis TES ether 18 (3.1 g, 80\% yield) as a pale yellow oil. $\mathrm{R}_{\mathrm{f}}=0.5(2.5 \%$ EtOAc/ hexanes); IR (film) $3301 \mathrm{~cm}^{-1} ;[\alpha]^{\mathrm{D}}{ }_{20}=+3.9\left(\mathrm{c}=1.36, \mathrm{CHCl}_{3}\right) ;{ }^{1} \mathrm{H} \mathrm{NMR}\left(\mathrm{CDCl}_{3}\right) \delta 3.76(\mathrm{dd}, \mathrm{J}=3.5 \mathrm{~Hz}$, $6.0 \mathrm{~Hz}, 1 \mathrm{H}$ ), 3.55 (A of ABX, J=7.0 Hz, 10.0 Hz, 1H), 3.44 (B of ABX, J = 7.0 Hz, 10.0 Hz, 1H), 2.64$2.59(\mathrm{~m}, 1 \mathrm{H}), 2.03(\mathrm{~d}, \mathrm{~J}=2.0 \mathrm{~Hz}, 1 \mathrm{H}), 1.89-1.82(\mathrm{~m}, 1 \mathrm{H}), 1.16(\mathrm{~d}, \mathrm{~J}=7.0 \mathrm{~Hz}, 3 \mathrm{H}), 0.99-0.94(\mathrm{~m}, 18 \mathrm{H})$, $0.87(\mathrm{~d}, \mathrm{~J}=7.0 \mathrm{~Hz}, 3 \mathrm{H}), 0.65(\mathrm{q}, \mathrm{J}=8.0 \mathrm{~Hz}, 6 \mathrm{H}), 0.59(\mathrm{q}, \mathrm{J}=8.0 \mathrm{~Hz}, 6 \mathrm{H}) ;{ }^{13} \mathrm{C} \mathrm{NMR}\left(\mathrm{CDCl}_{3}\right) \delta 87.71$, $74.80,69.53,65.60,39.03,31.52,16.46,11.02,7.05,6.78,5.42,4.46$. 


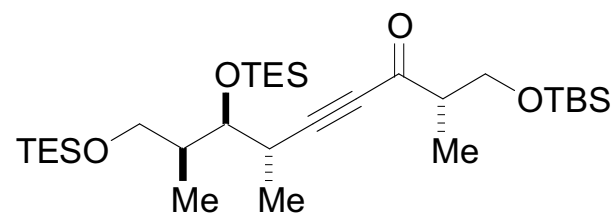

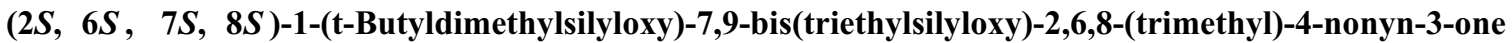
(21)

Alkyne $18(0.93 \mathrm{~g}, 2.5 \mathrm{mmol})$ was taken up in $19 \mathrm{~mL}$ of THF and chilled to $-78{ }^{\circ} \mathrm{C}$. $\mathrm{BuLi}(1.0 \mathrm{~mL}$, 2.5 $\mathrm{M}$ in hexanes) was added slowly. After $5 \mathrm{~min}$, the mixture was warmed to $0{ }^{\circ} \mathrm{C}$ and stirred for $40 \mathrm{~min}$. The mixture was then chilled to $-78{ }^{\circ} \mathrm{C}$, and amide $20(0.50 \mathrm{~g}, 1.9 \mathrm{mmol})$ in $3 \mathrm{~mL}$ of $\mathrm{THF}$ was added slowly. After $5 \mathrm{~min}$, the solution was warmed to $0{ }^{\circ} \mathrm{C}$ and stirred for $30 \mathrm{~min}$. The reaction was quenched with aq $\mathrm{NH}_{4} \mathrm{Cl}$ and the mixture was partitioned in a separatory funnel. The aqueous portion was extracted three times with ether and the combined organic extracts were washed with brine and dried over $\mathrm{MgSO}_{4}$. Filtration and concentration under reduced pressure, followed by flash chromatography on silica gel (100\% hexanes to $1 \%$ EtOAc/ hexanes), afforded ynone 21 (1.1 g, 100\% yield) as a yellow oil. $\mathrm{R}_{\mathrm{f}}=0.25(5 \%$ EtOAc/ hexanes); $[\alpha]^{\mathrm{D}}{ }_{20}=+2.1\left(\mathrm{c}=1.80, \mathrm{CHCl}_{3}\right)$; IR (film) 2209, $1693 \mathrm{~cm}^{-1} ;{ }^{1} \mathrm{H}$ NMR $\left(\mathrm{CDCl}_{3}\right) \delta 3.90(\mathrm{dd}$, $\mathrm{J}=6.5 \mathrm{~Hz}, 10.0 \mathrm{~Hz}, 1 \mathrm{H}), 3.81(\mathrm{t}, \mathrm{J}=5.0 \mathrm{~Hz}, 1 \mathrm{H}), 3.72(\mathrm{dd}, \mathrm{J}=6.0 \mathrm{~Hz}, 10.0 \mathrm{~Hz}, 1 \mathrm{H}), 3.52(\mathrm{~A}$ of $\mathrm{ABX}, \mathrm{J}=$ $7.0 \mathrm{~Hz}, 10.0 \mathrm{~Hz}, 1 \mathrm{H}), 3.45$ (B of ABX, J = 6.0 Hz, 10.0 Hz, 1H), 2.85-2.80 (m, 1H), 2.76-2.69 (m, 1H), 1.86-1.82 (m, 1H), $1.23(\mathrm{~d}, \mathrm{~J}=7.5 \mathrm{~Hz}, 3 \mathrm{H}), 1.16(\mathrm{~d}, \mathrm{~J}=7.0 \mathrm{~Hz}, 3 \mathrm{H}), 0.97(\mathrm{t}, \mathrm{J}=8.0 \mathrm{~Hz}, 9 \mathrm{H}), 0.95(\mathrm{t}, \mathrm{J}=$ $8.0 \mathrm{~Hz}, 9 \mathrm{H}), 0.89$ (d, J = 7.0 Hz, 3H), 0.88 (s, 9H), 0.64 (q, J = 8.0 Hz, 6H), 0.58 (q, J = 8.0 Hz, 6H), 0.04 $(\mathrm{d}, \mathrm{J}=1.5 \mathrm{~Hz}, 6 \mathrm{H}) ;{ }^{13} \mathrm{C} \mathrm{NMR}\left(\mathrm{CDCl}_{3}\right) \delta 190.13,97.55,81.38,74.72,65.25,64.51,51.26,39.76,32.15$, $25.78,18.21,17.26,12.76,11.49,7.02,6.77,6.74,5.37,4.40,-5.51$.

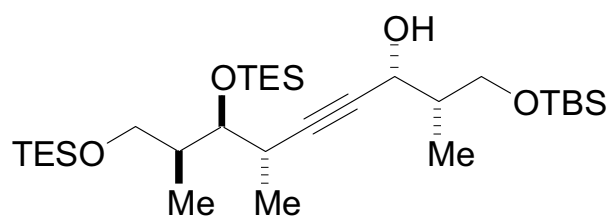

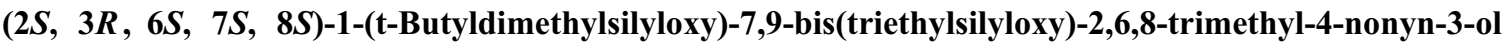
(22)

Ynone 21 (1.1 g, $1.9 \mathrm{mmol})$ was taken up in $19 \mathrm{~mL}$ of i-PrOH. Catalyst 14 (0.056 g, $0.096 \mathrm{mmol})$ was added in one portion, and the solution was stirred overnight. The solvent was removed under vacuum, and the crude residue was purified by flash chromatography on silica gel (2.5\% EtOAc/ hexanes), affording propargylic alcohol $22\left(0.93 \mathrm{~g}, 85 \%\right.$ yield) as a yellow oil. $\mathrm{R}_{\mathrm{f}}=0.20$ (5\% EtOAc/ hexanes); $[\alpha]_{20}^{\mathrm{D}}=+10.2\left(\mathrm{c}=1.36, \mathrm{CHCl}_{3}\right)$; IR (film) 3501, $2235 \mathrm{~cm}^{-1} ;{ }^{1} \mathrm{H} \mathrm{NMR}\left(\mathrm{CDCl}_{3}\right) \delta 4.44(\mathrm{~s}, 1 \mathrm{H}), 3.85(\mathrm{t}, \mathrm{J}=$ $9.5 \mathrm{~Hz}, 1 \mathrm{H}), 3.75(\mathrm{t}, \mathrm{J}=4.5 \mathrm{~Hz}, 1 \mathrm{H}), 3.63(\mathrm{dd}, \mathrm{J}=4.5 \mathrm{~Hz}, 10.0 \mathrm{~Hz}, 1 \mathrm{H}), 3.53$ (A of ABX, J = 6.5 Hz, 9.5 $\mathrm{Hz}, 1 \mathrm{H}), 3.43$ (B of ABX, J = 6.5 Hz, 10.0 Hz, 1H), 2.70-2.64 (m ,1H), 2.12-2.04 (m, 1H), 1.92-1.84 (m, $1 \mathrm{H}), 1.70(\mathrm{~d}, \mathrm{~J}=7.0 \mathrm{~Hz}, 3 \mathrm{H}), 0.99-0.94(\mathrm{~m}, 18 \mathrm{H}), 0.90(\mathrm{~s}, 9 \mathrm{H}), 0.88(\mathrm{~d}, \mathrm{~J}=7.0 \mathrm{~Hz}, 3 \mathrm{H}), 0.87(\mathrm{~d}, \mathrm{~J}=7.0$ $\mathrm{Hz}, 3 \mathrm{H}), 0.64$ (q, J = 7.0 Hz, 6H), 0.58 (q, J = $7.5 \mathrm{~Hz}, 6 \mathrm{H}), 0.09(\mathrm{~s}, 3 \mathrm{H}), 0.08(\mathrm{~s}, 3 \mathrm{H}) ;{ }^{13} \mathrm{C} \mathrm{NMR}\left(\mathrm{CDCl}_{3}\right) \delta$ 
$88.83,80.61,74.95,67.14,66.68,65.69,40.19,39.32,31.83,25.82,18.12,17.84,12.36,11.56,7.06,6.79$, $5.40,4.43,-5.59,-5.65$.

\section{References}

1) White, J. A.; Hanselmann, R.; Jackson, R. W.; Porter, W. J.; Okba, Y.; Tiller, T.; Wang, S. J. Org. Chem., 2001, 66, 5217.

2) Marshall, J. A.; Schaaf, G. M. J. Org. Chem., 2001, 66, 7825. 


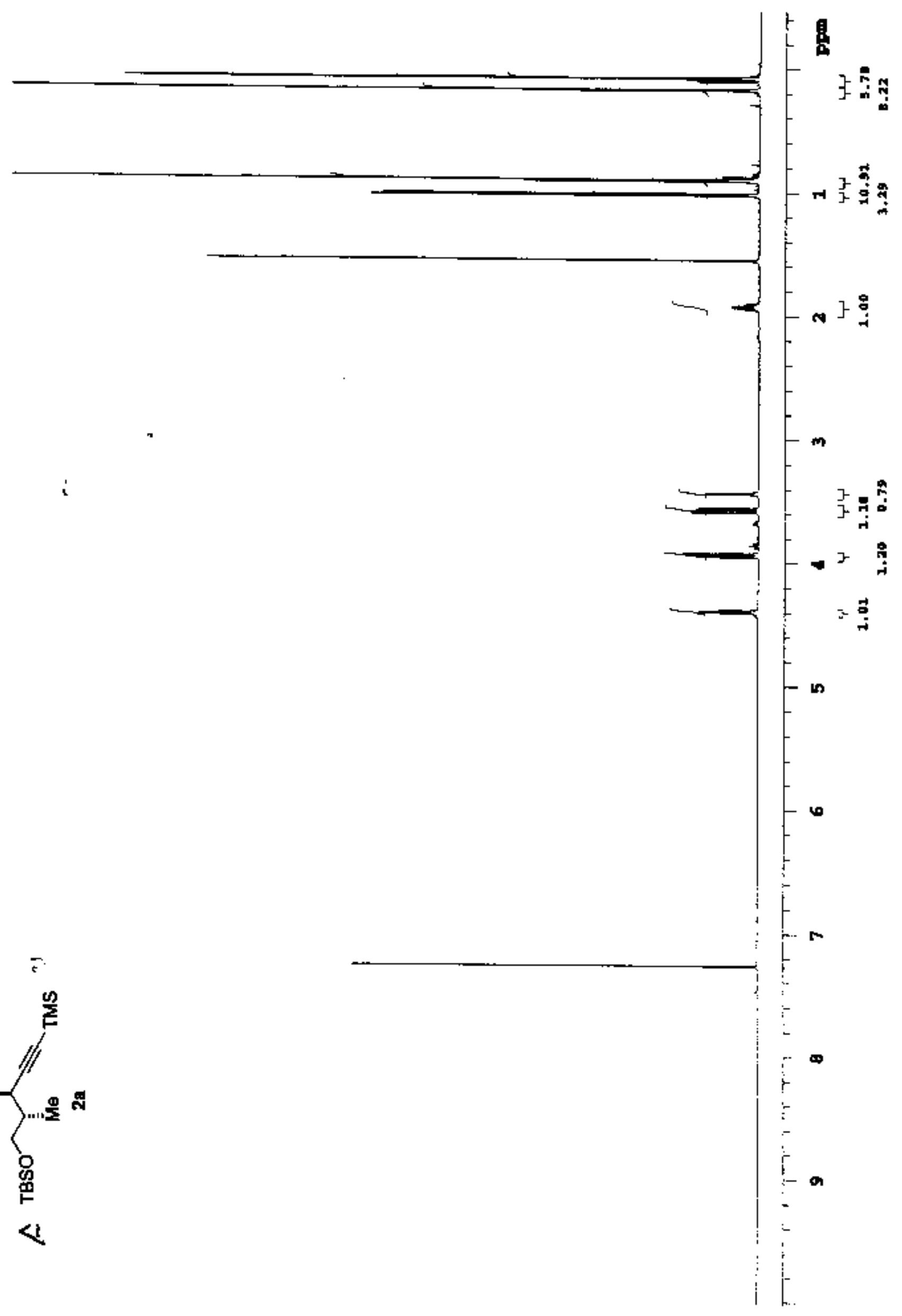




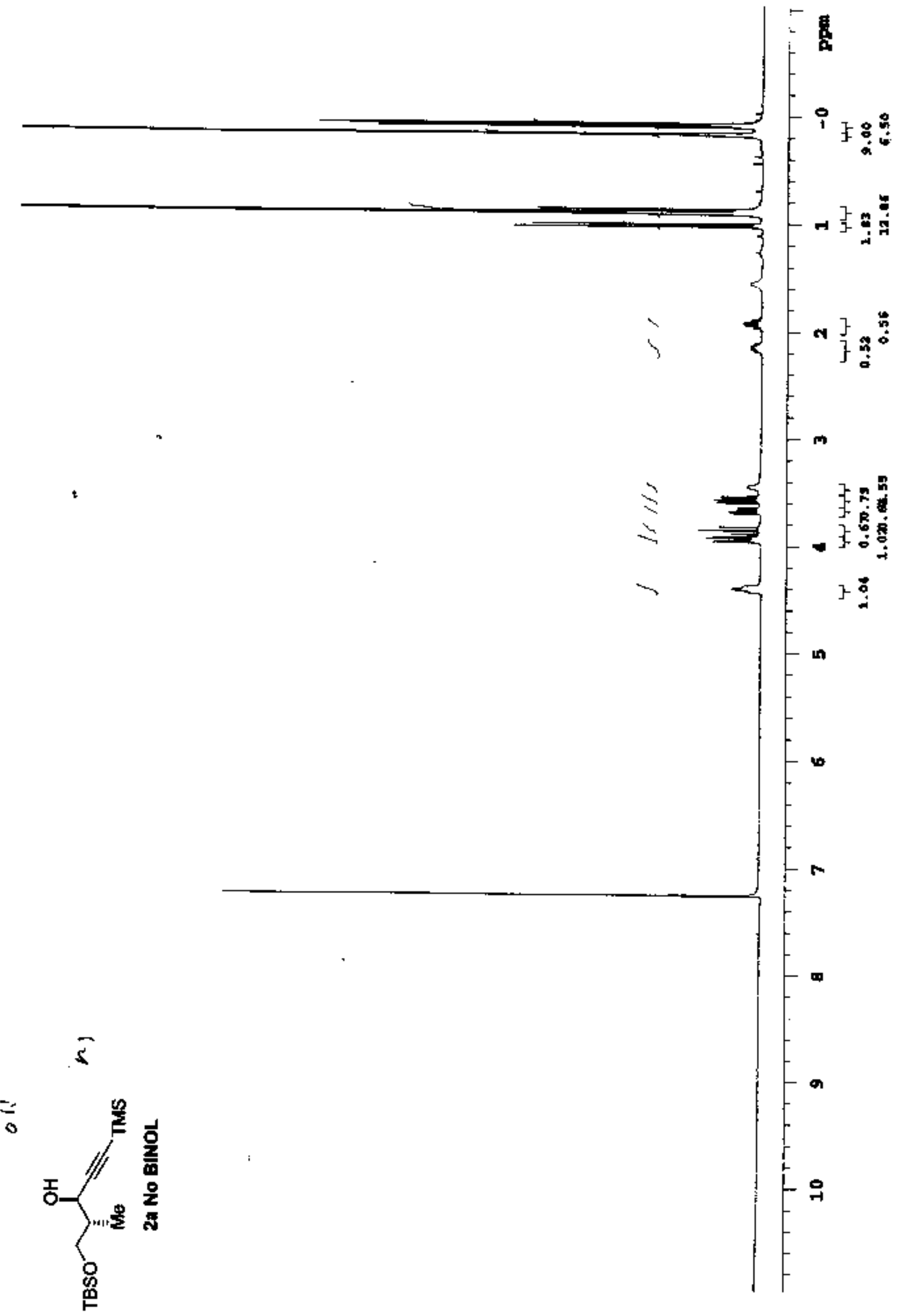




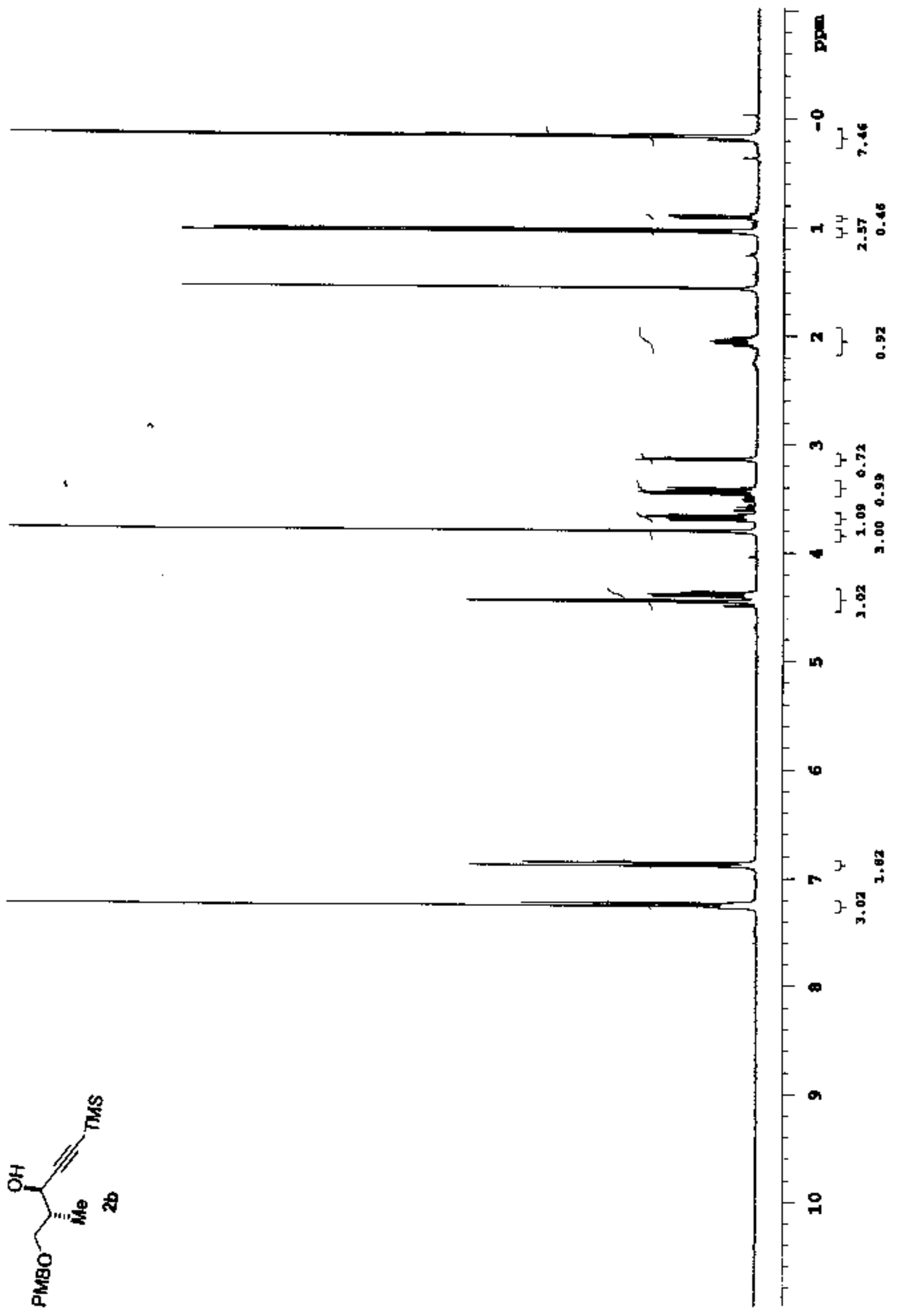




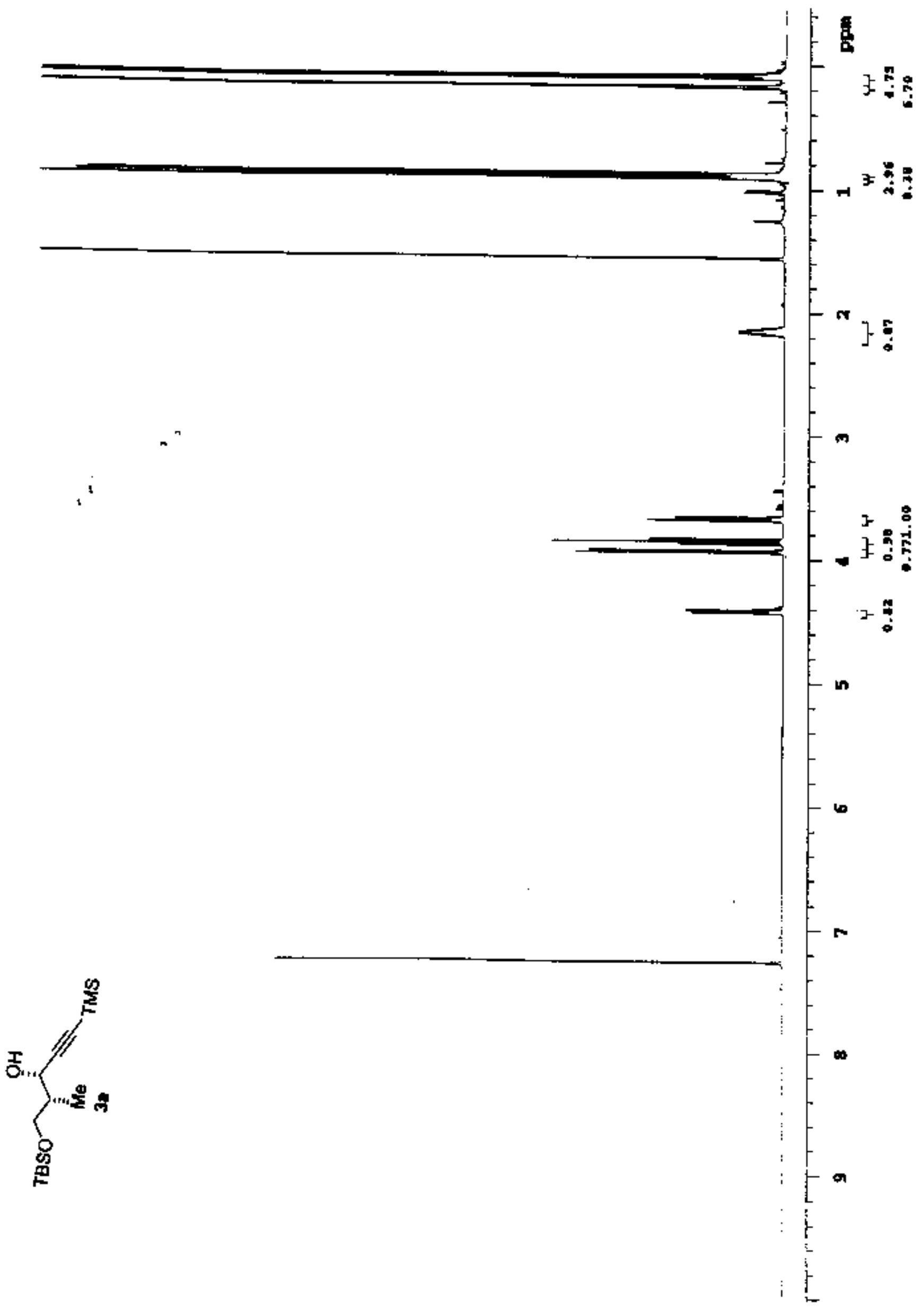




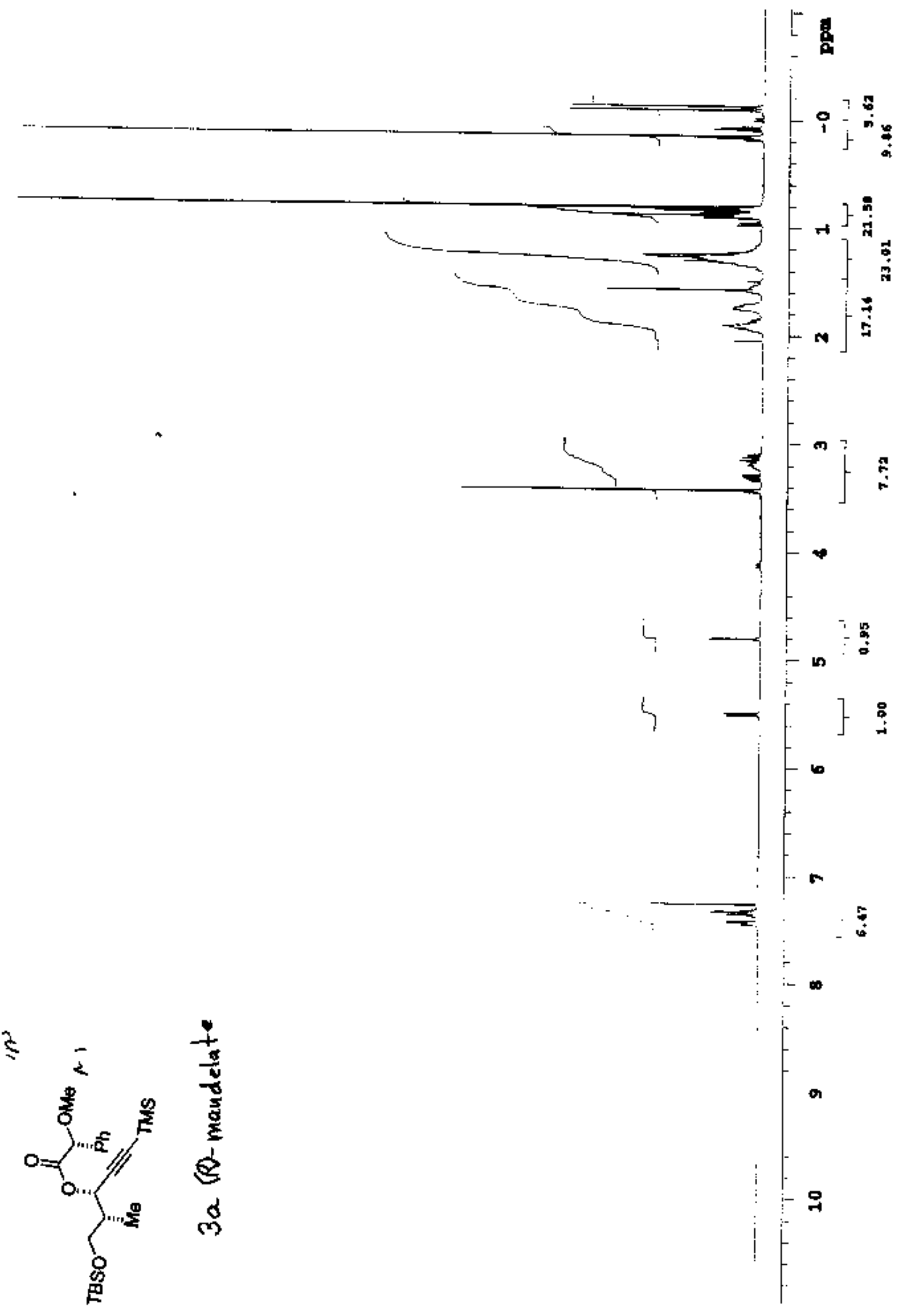




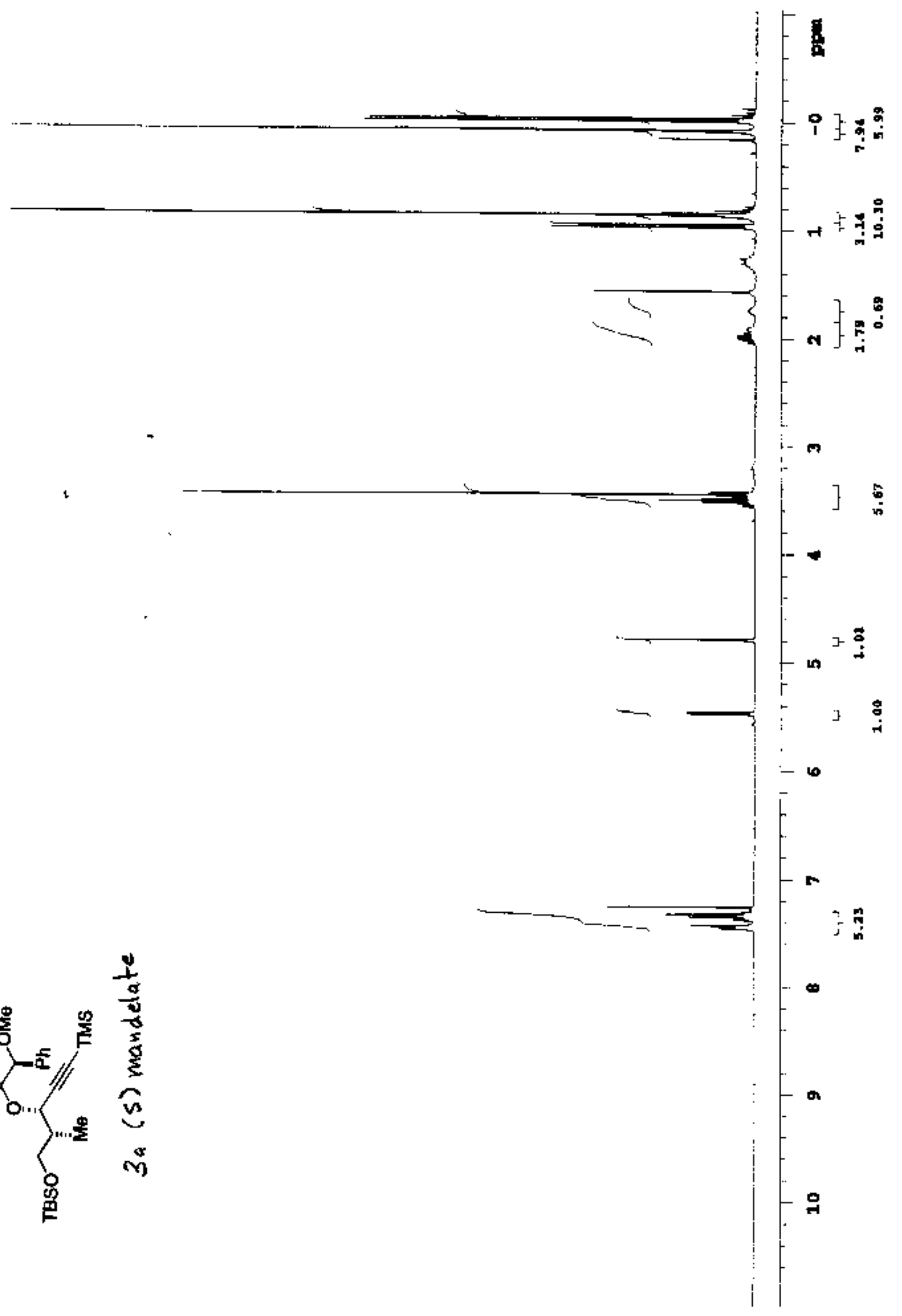




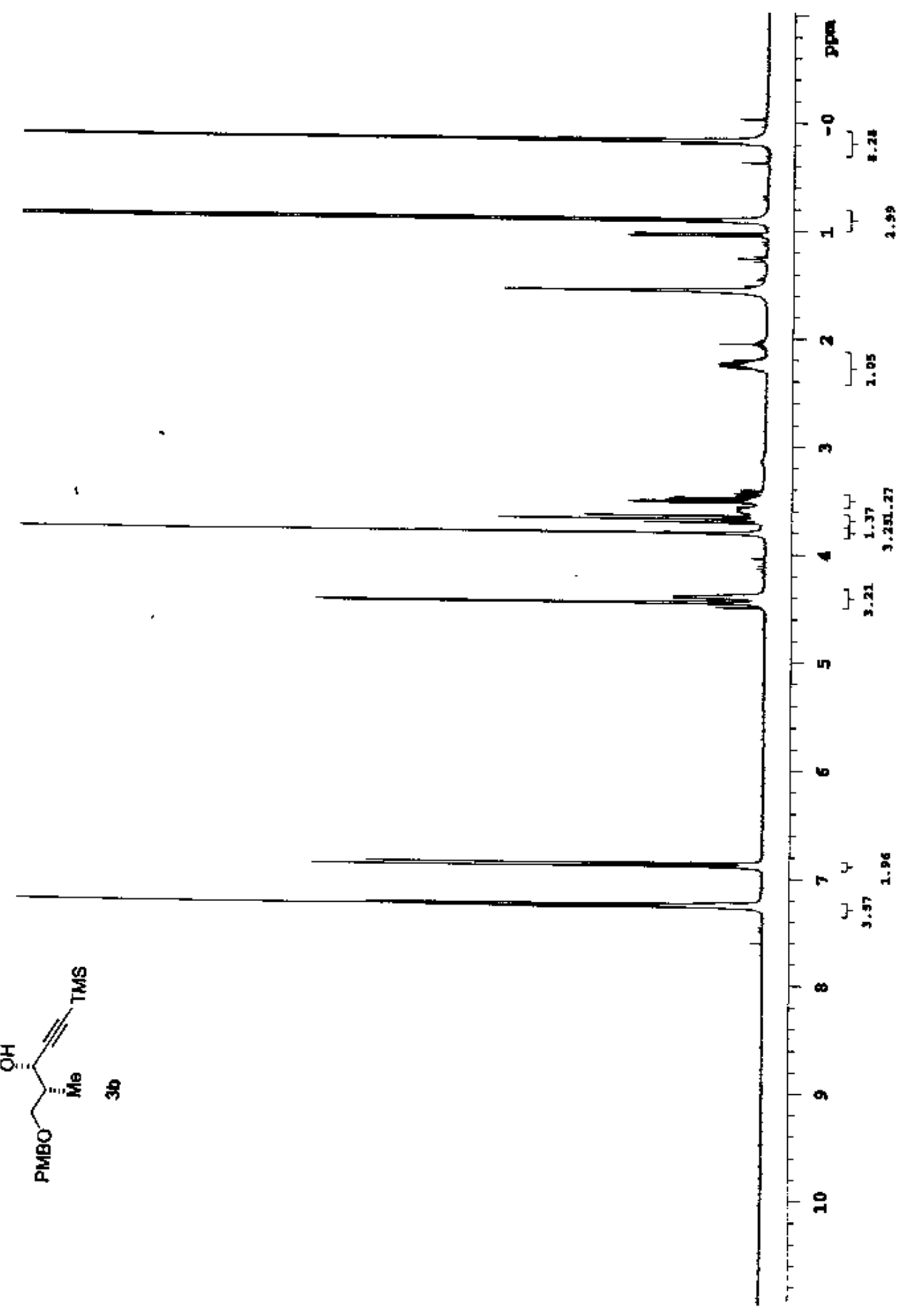




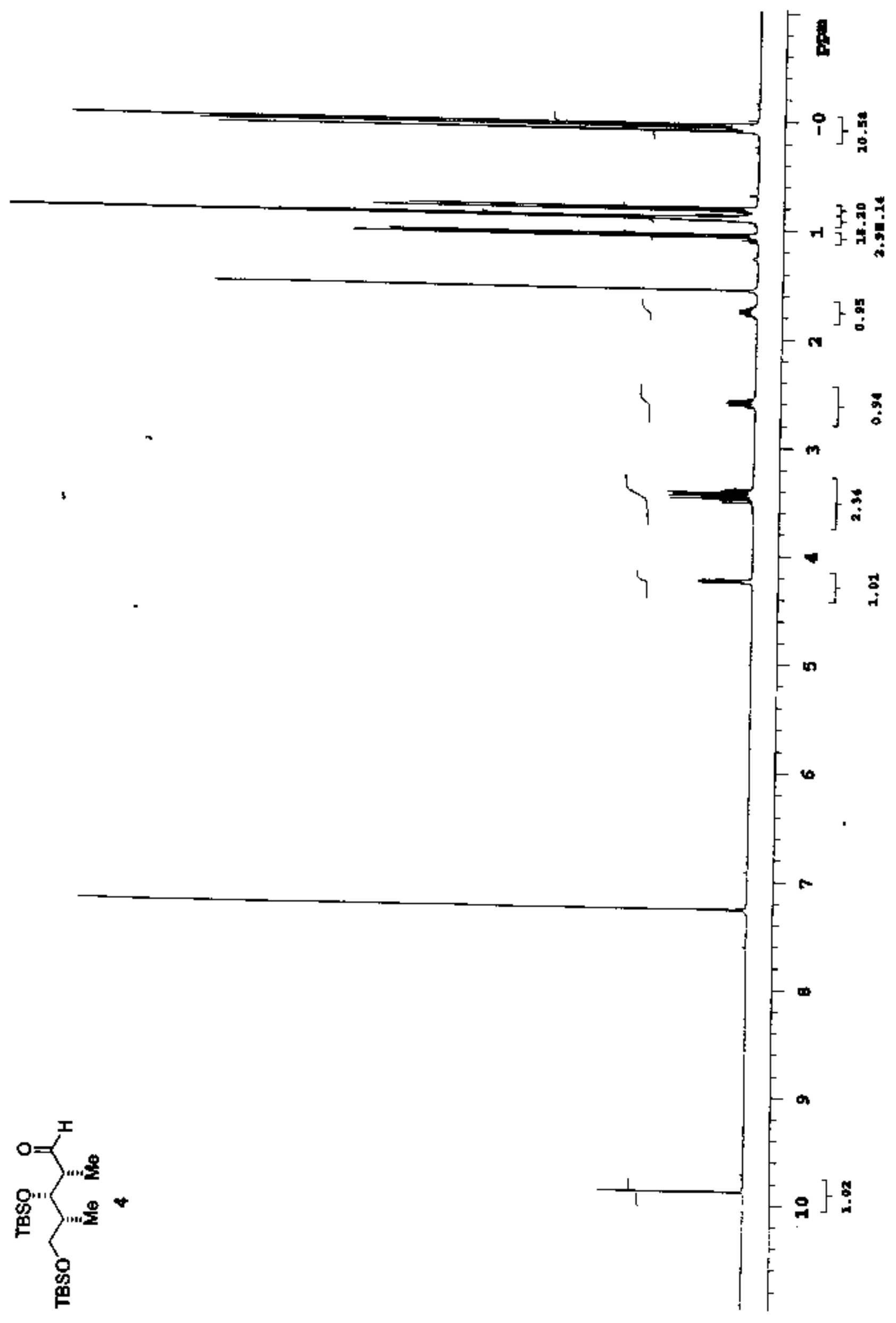




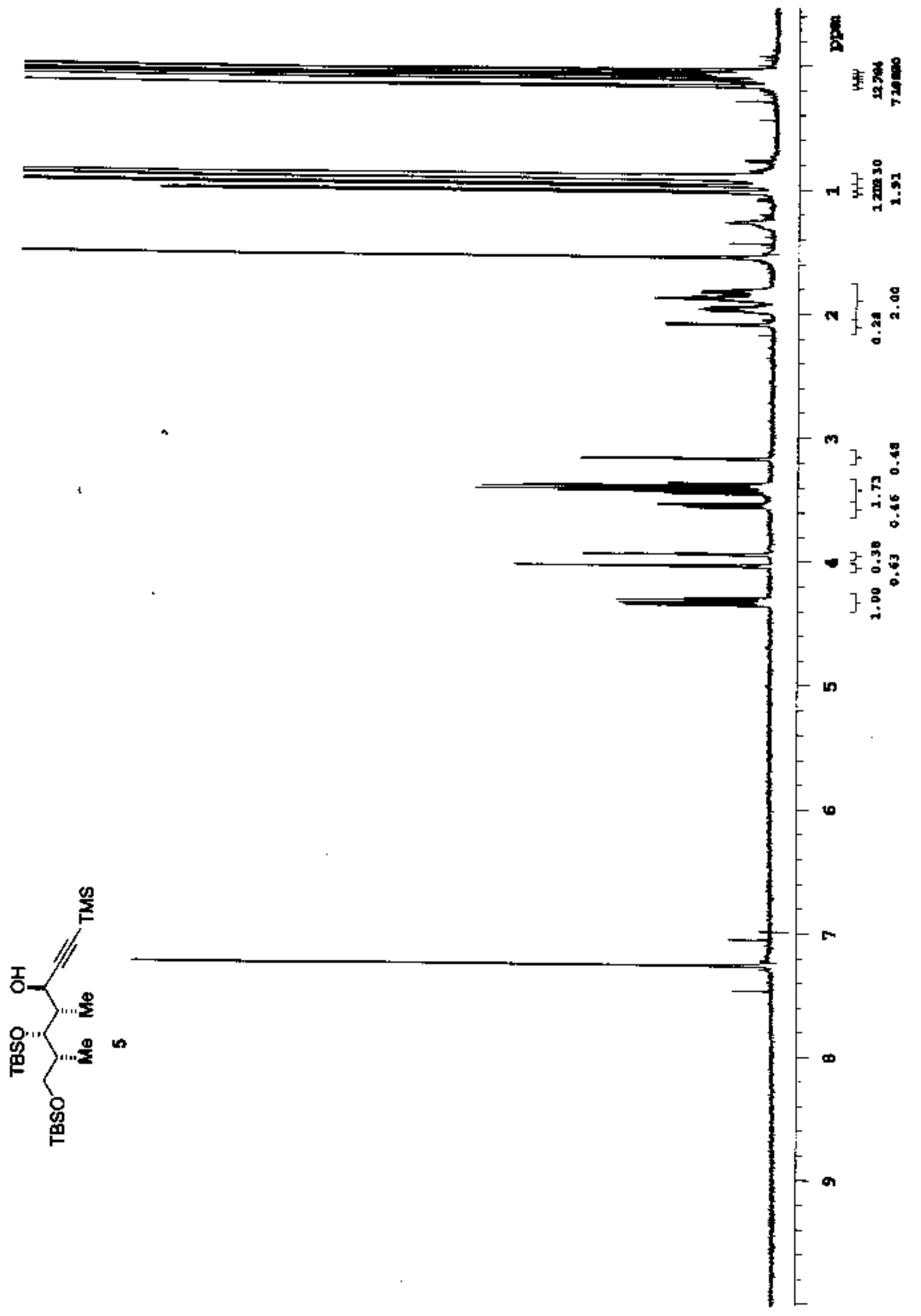




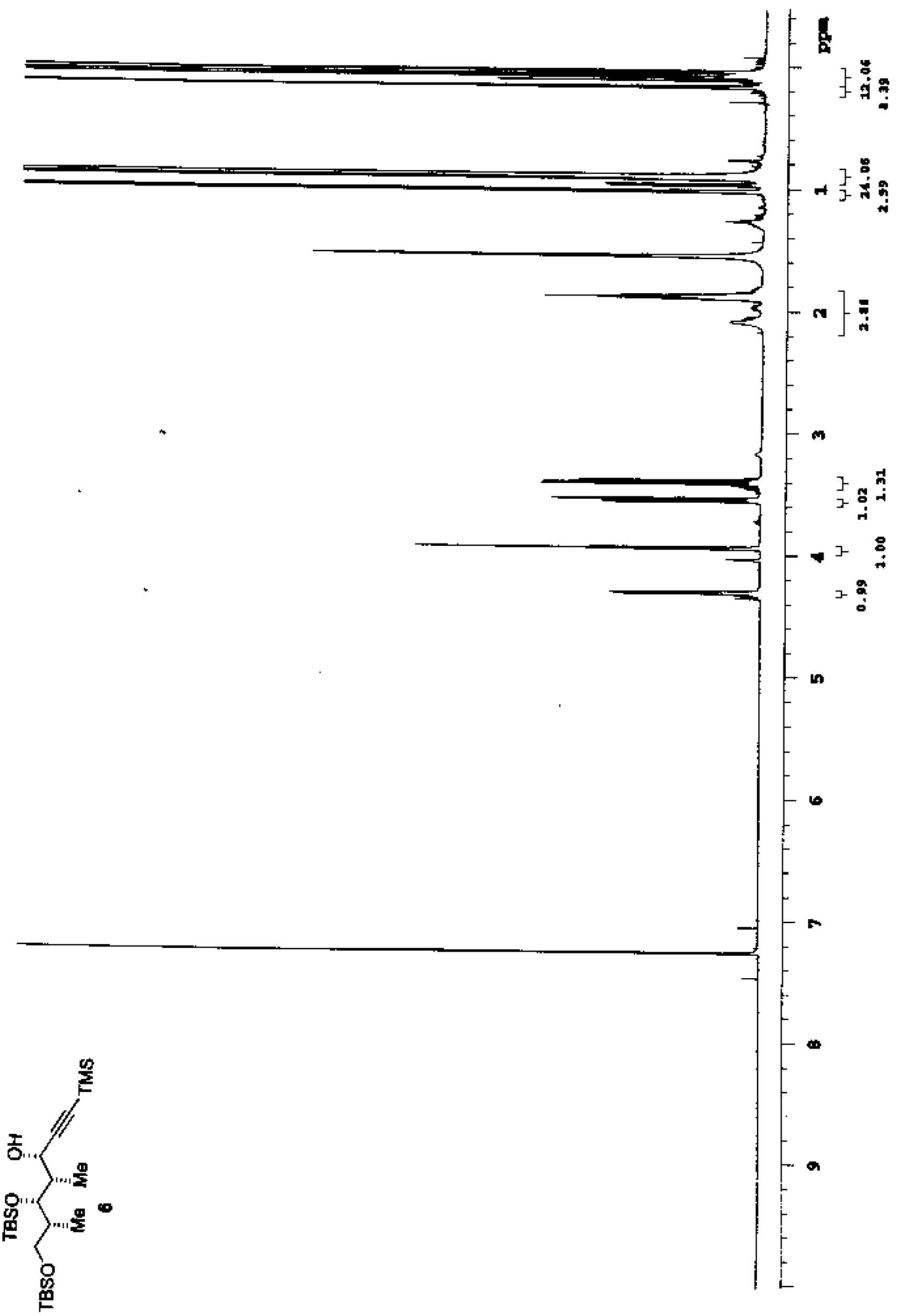




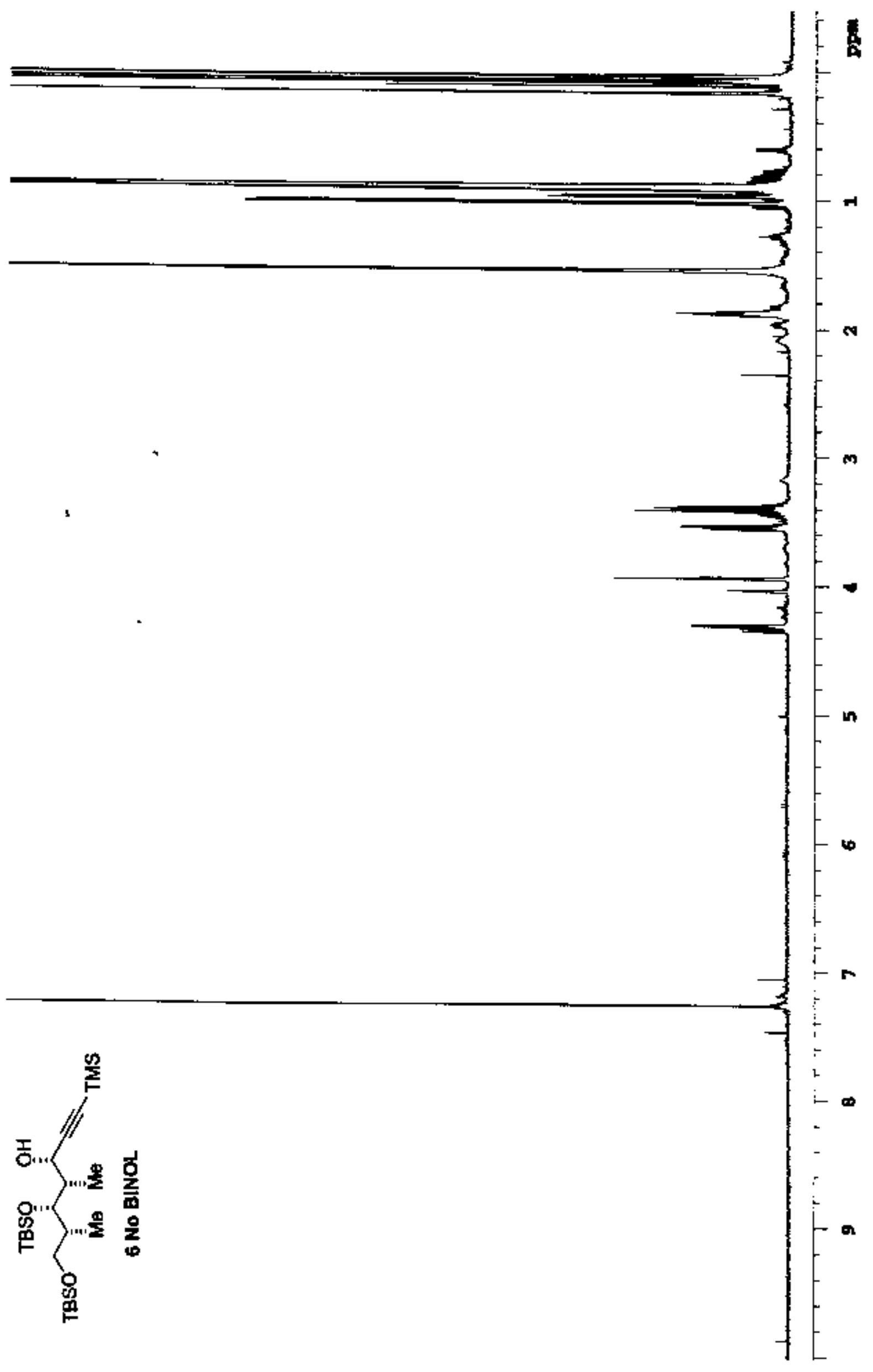




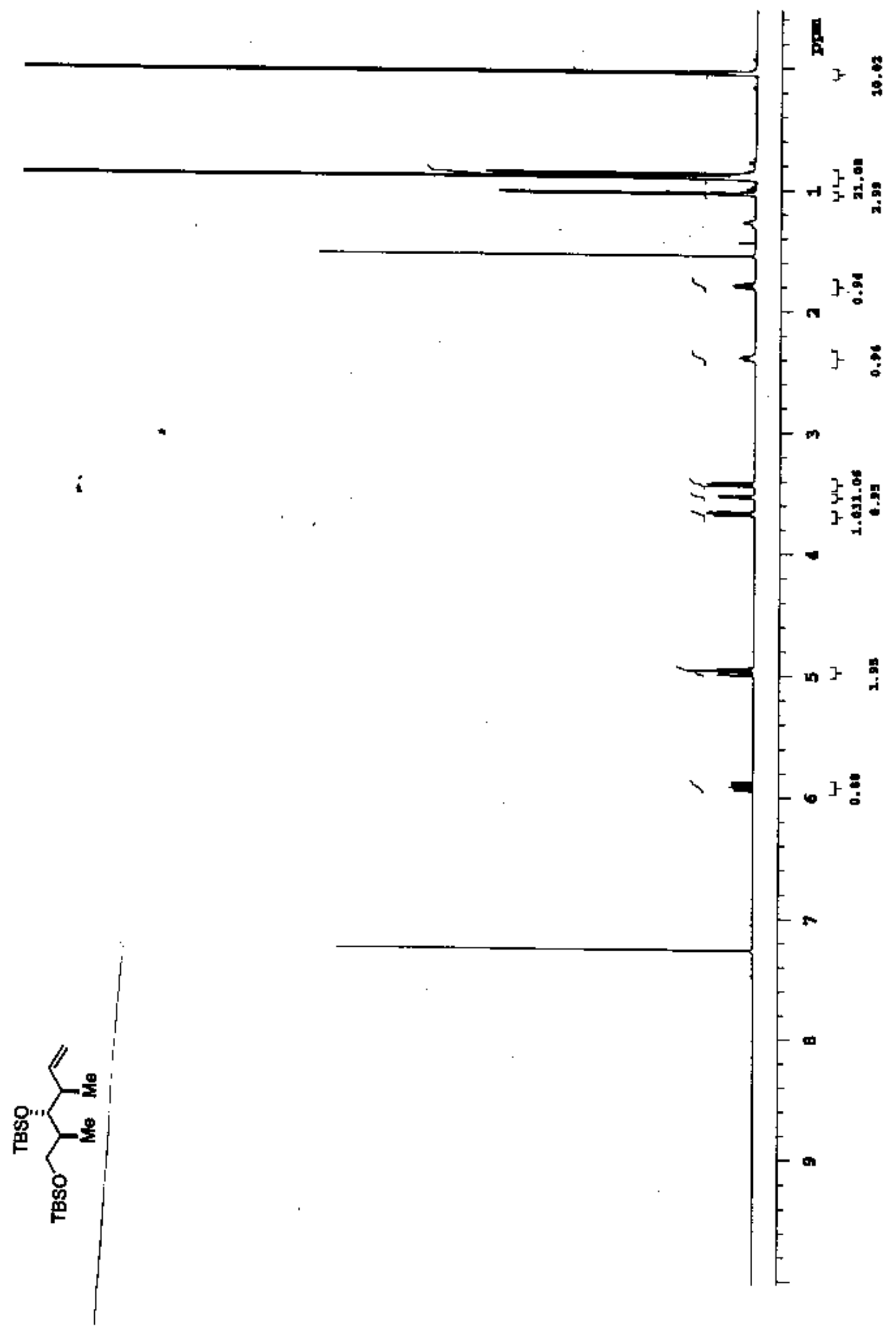




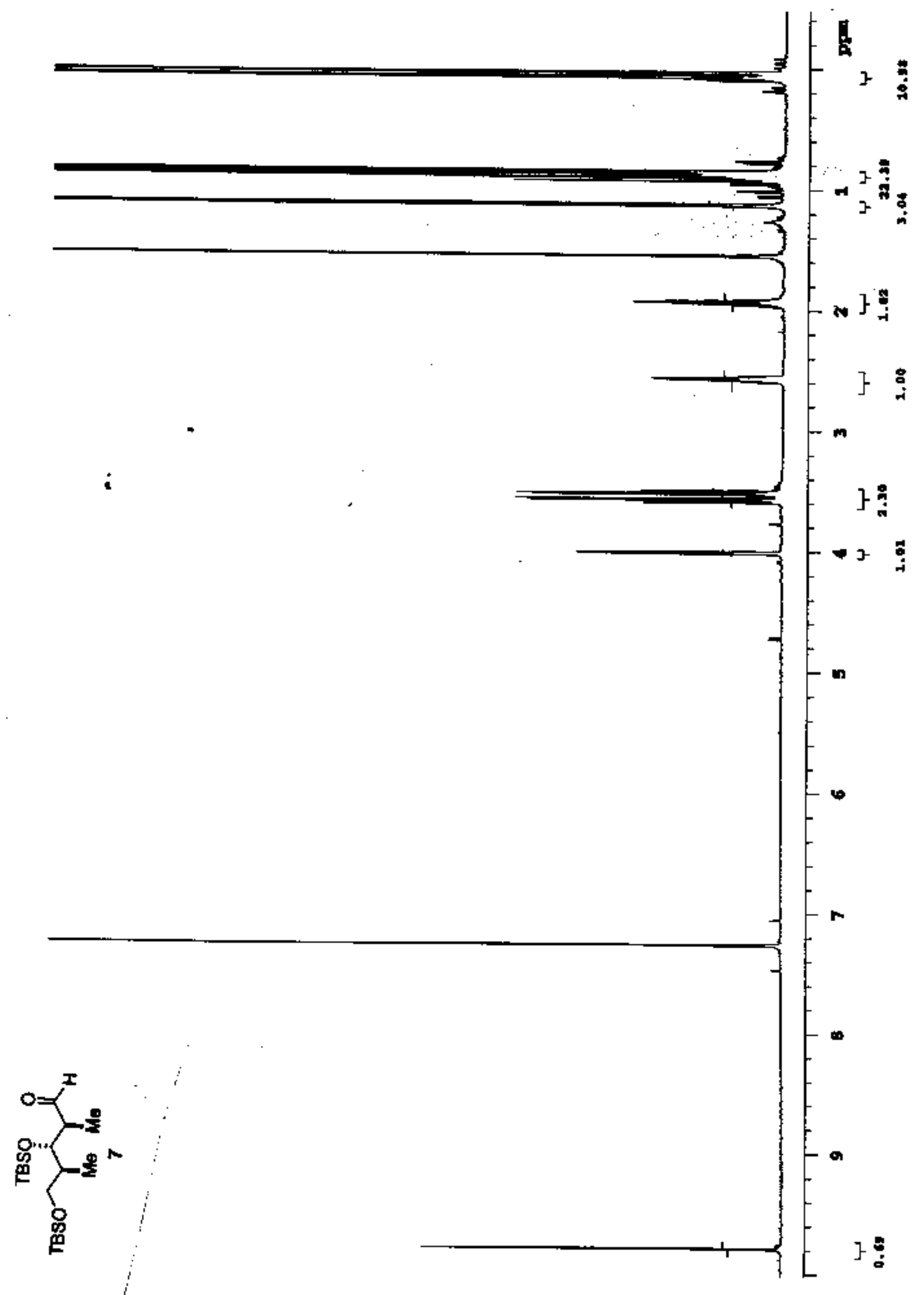




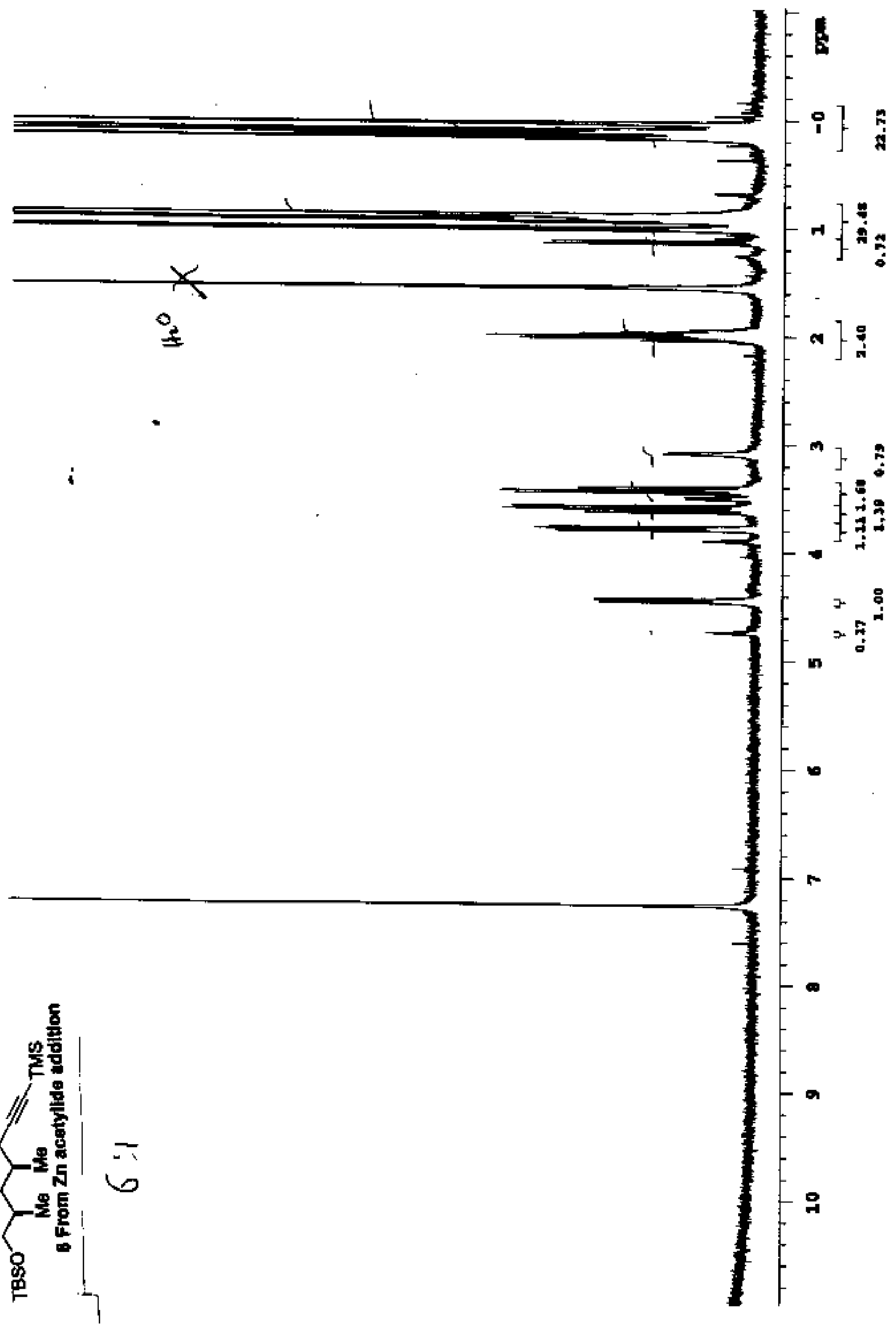




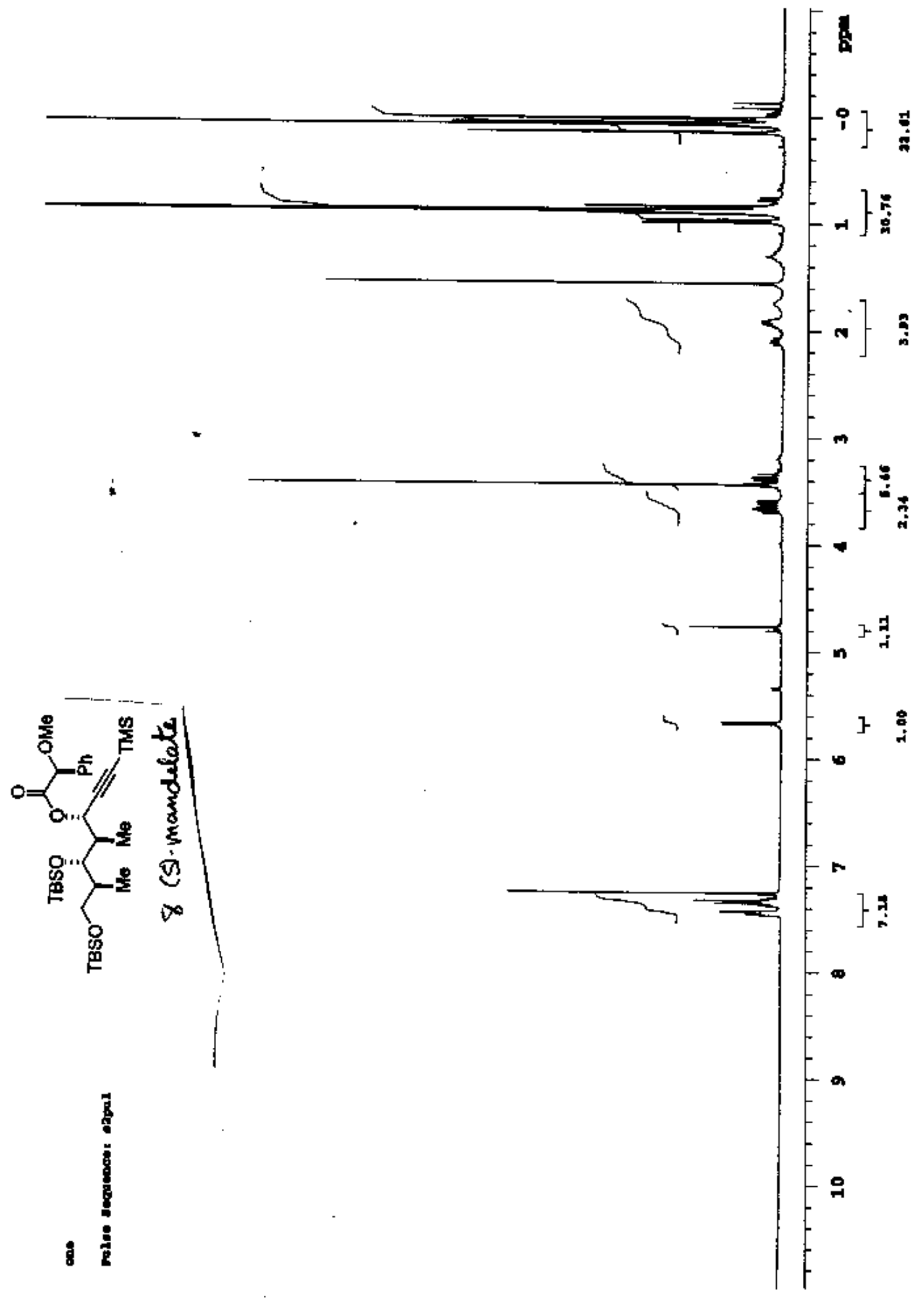




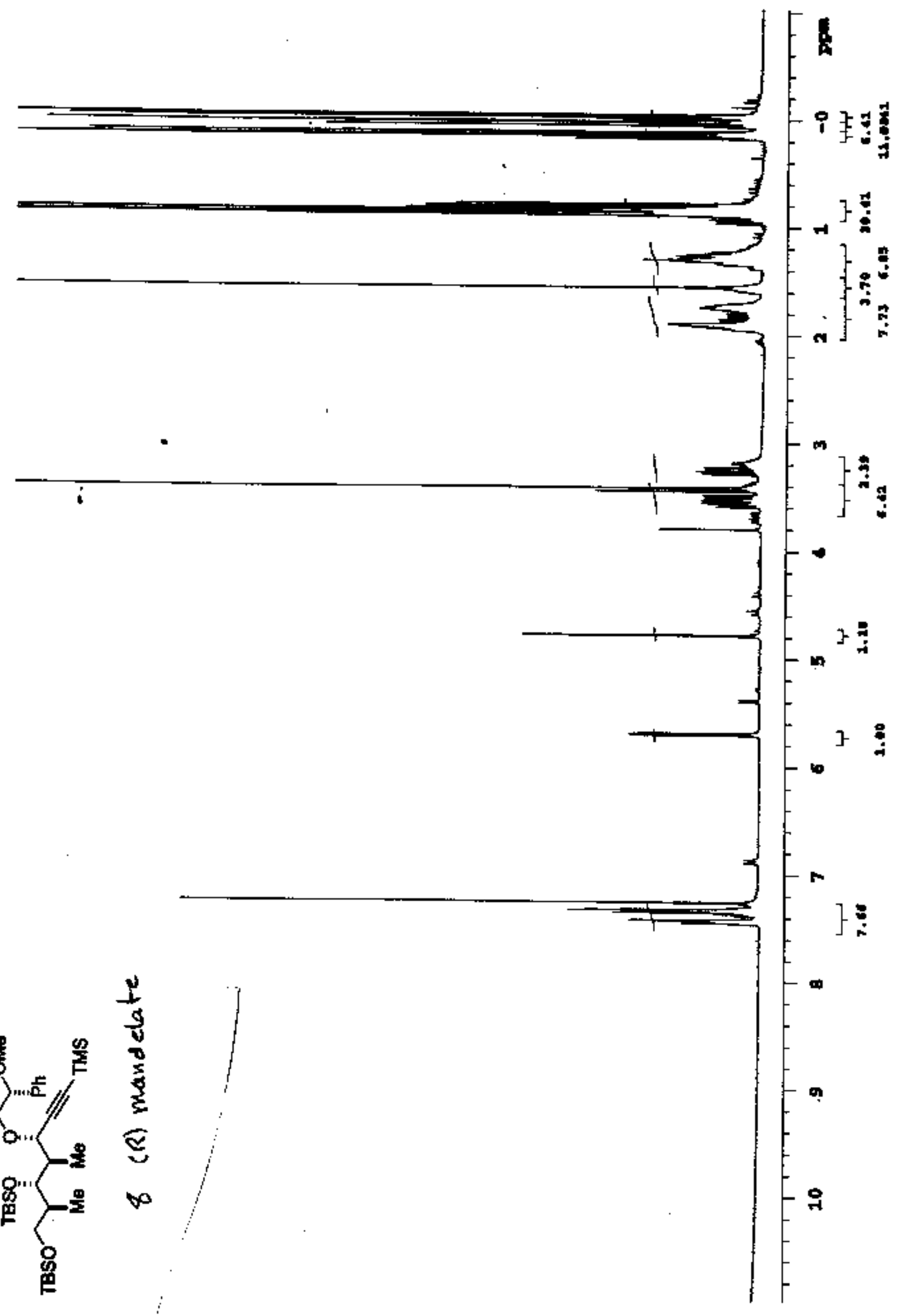




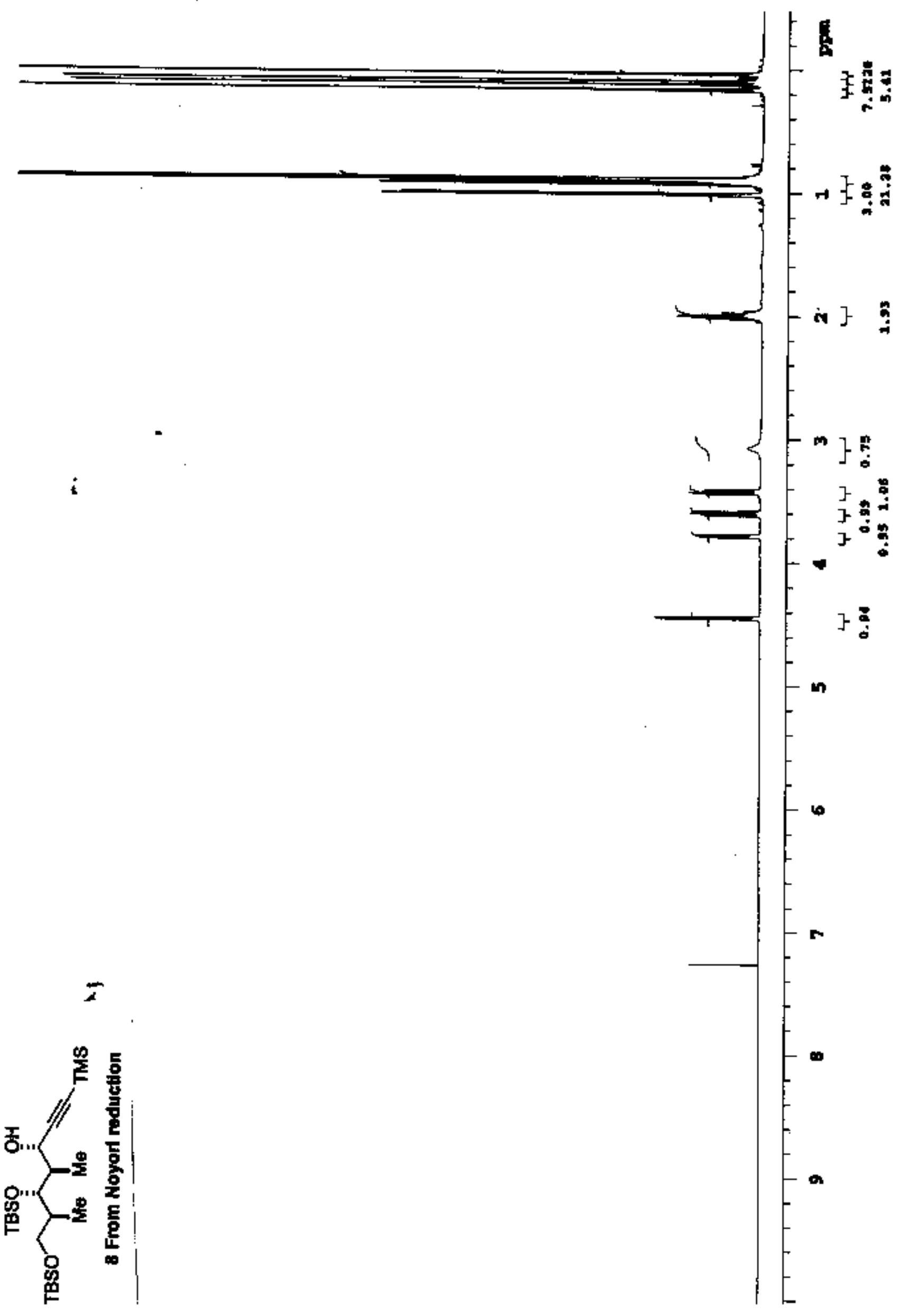




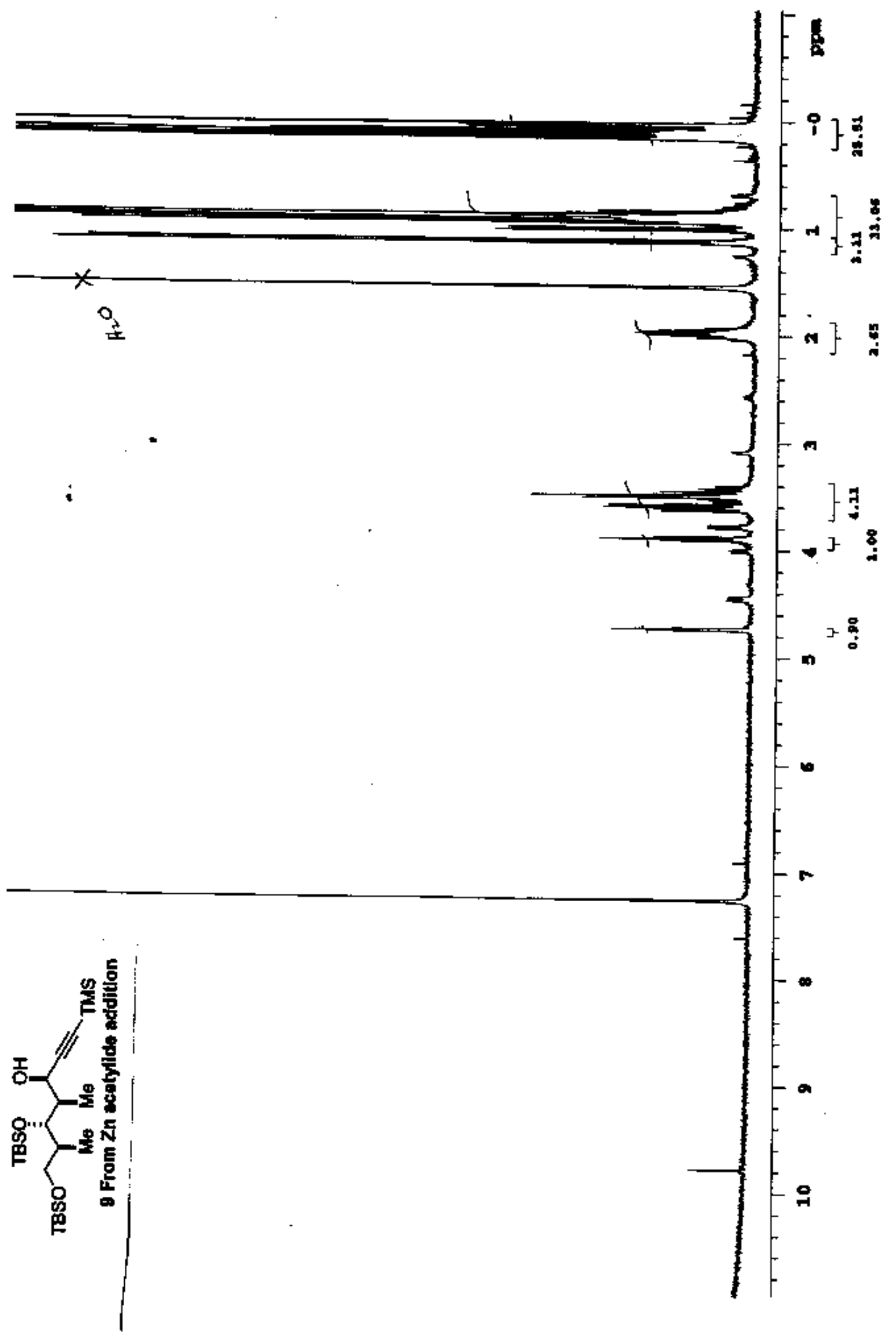




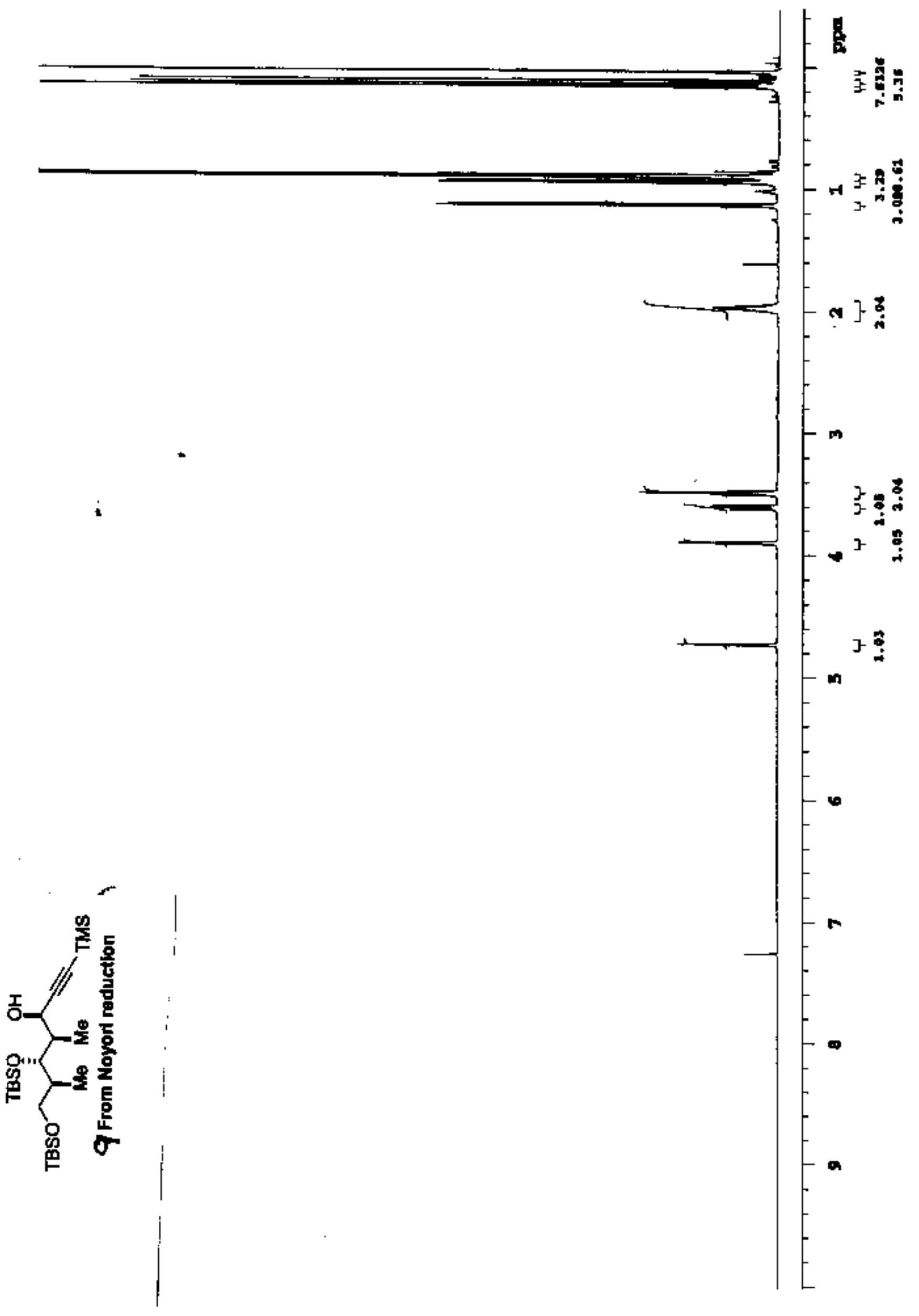




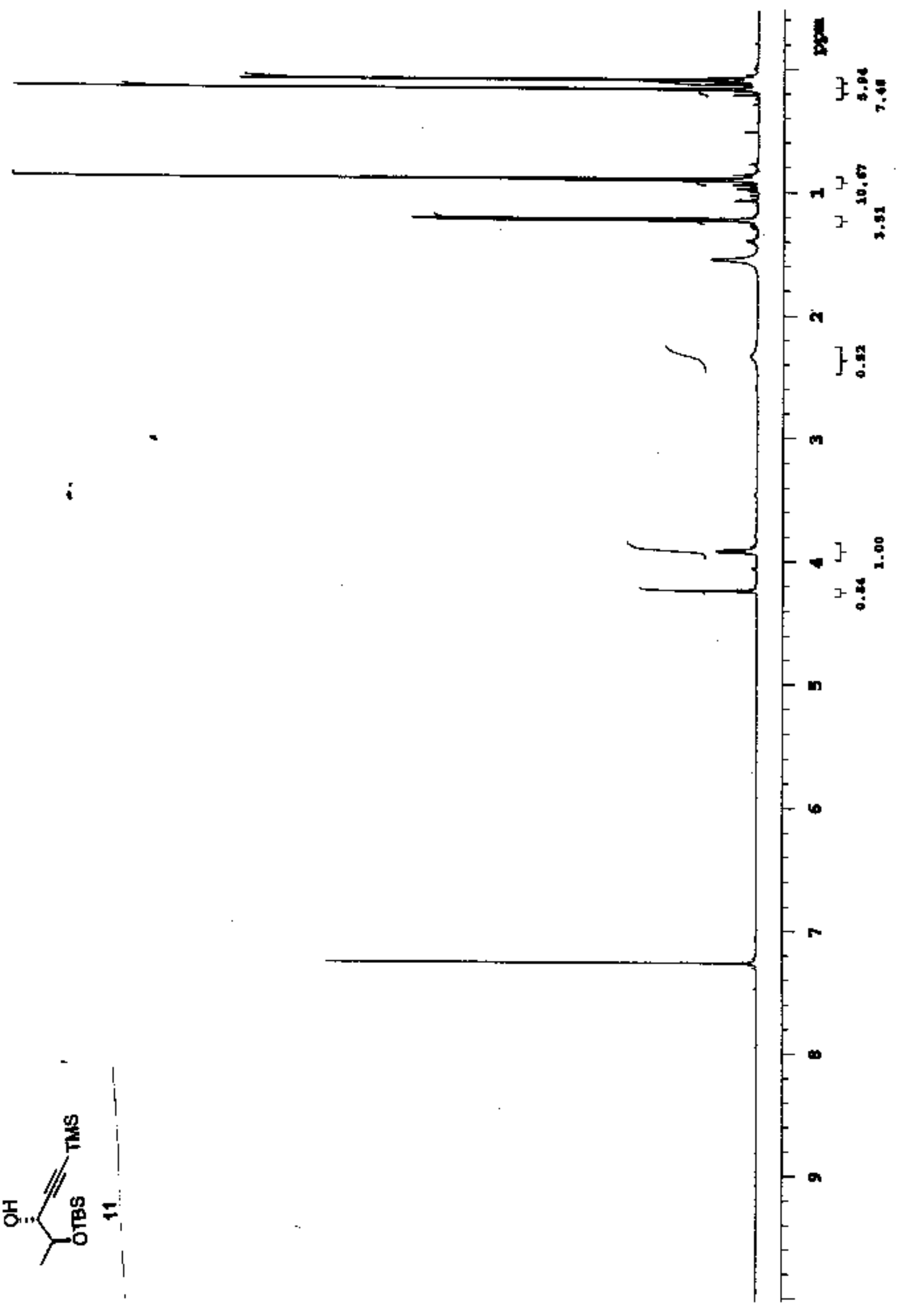




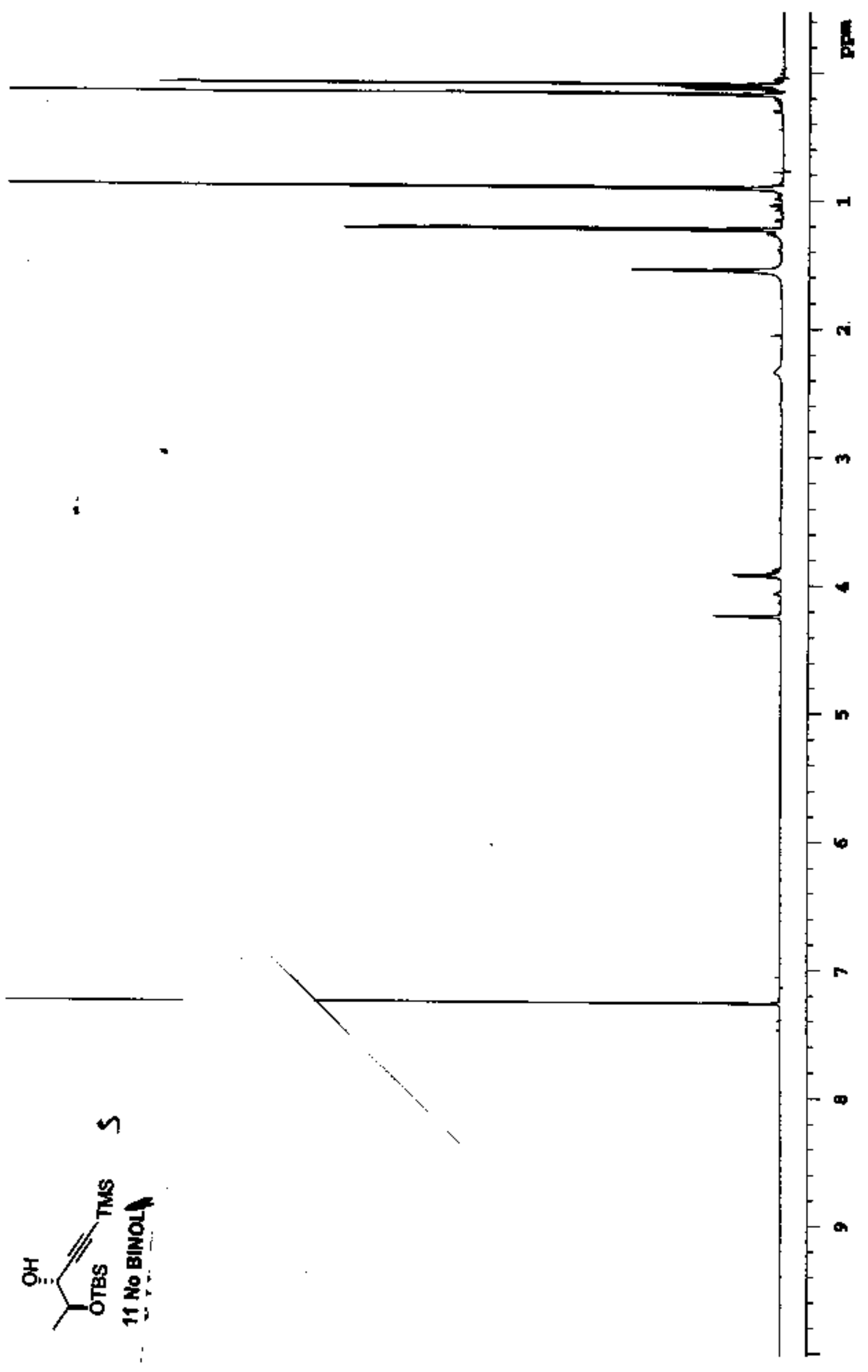




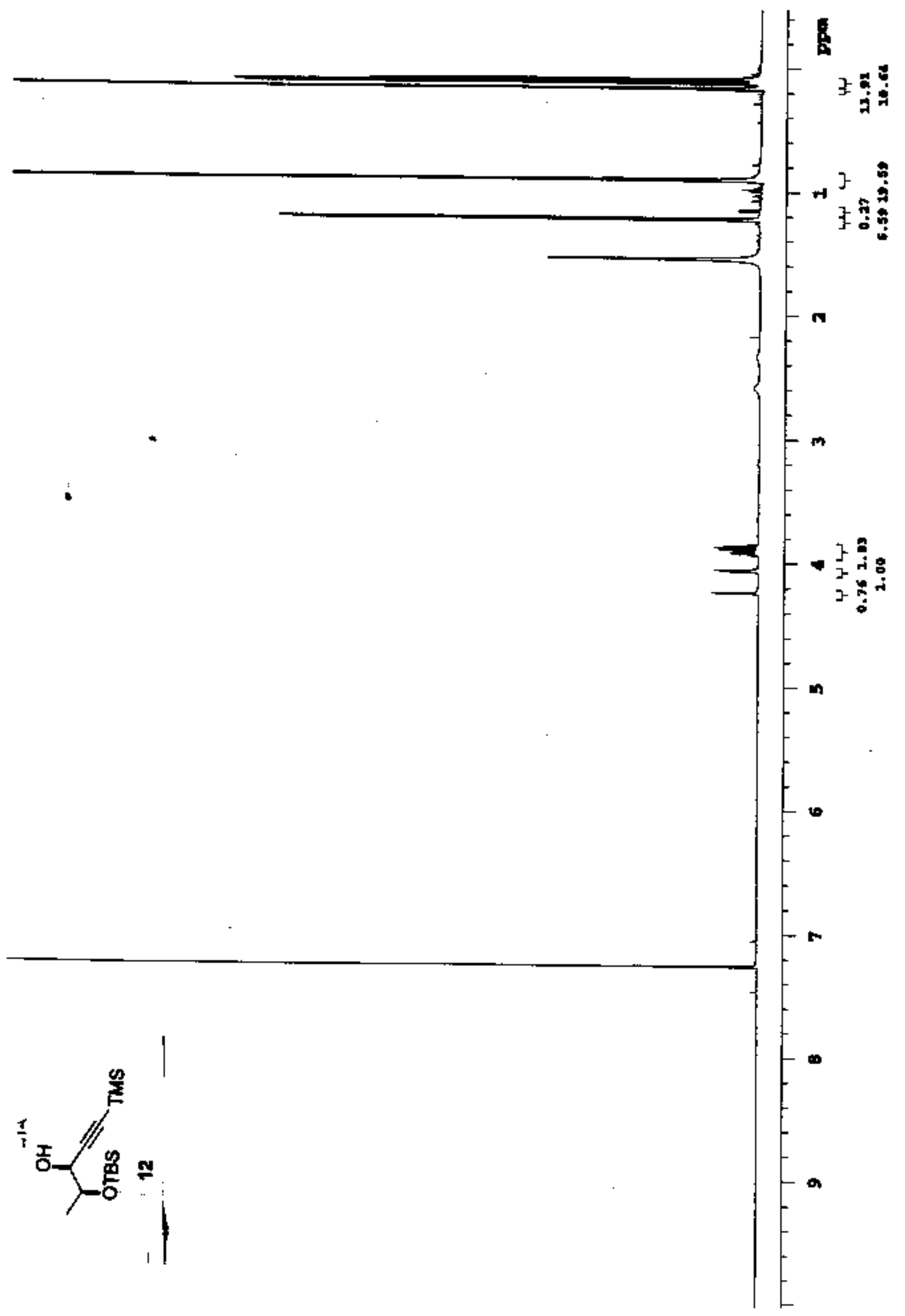




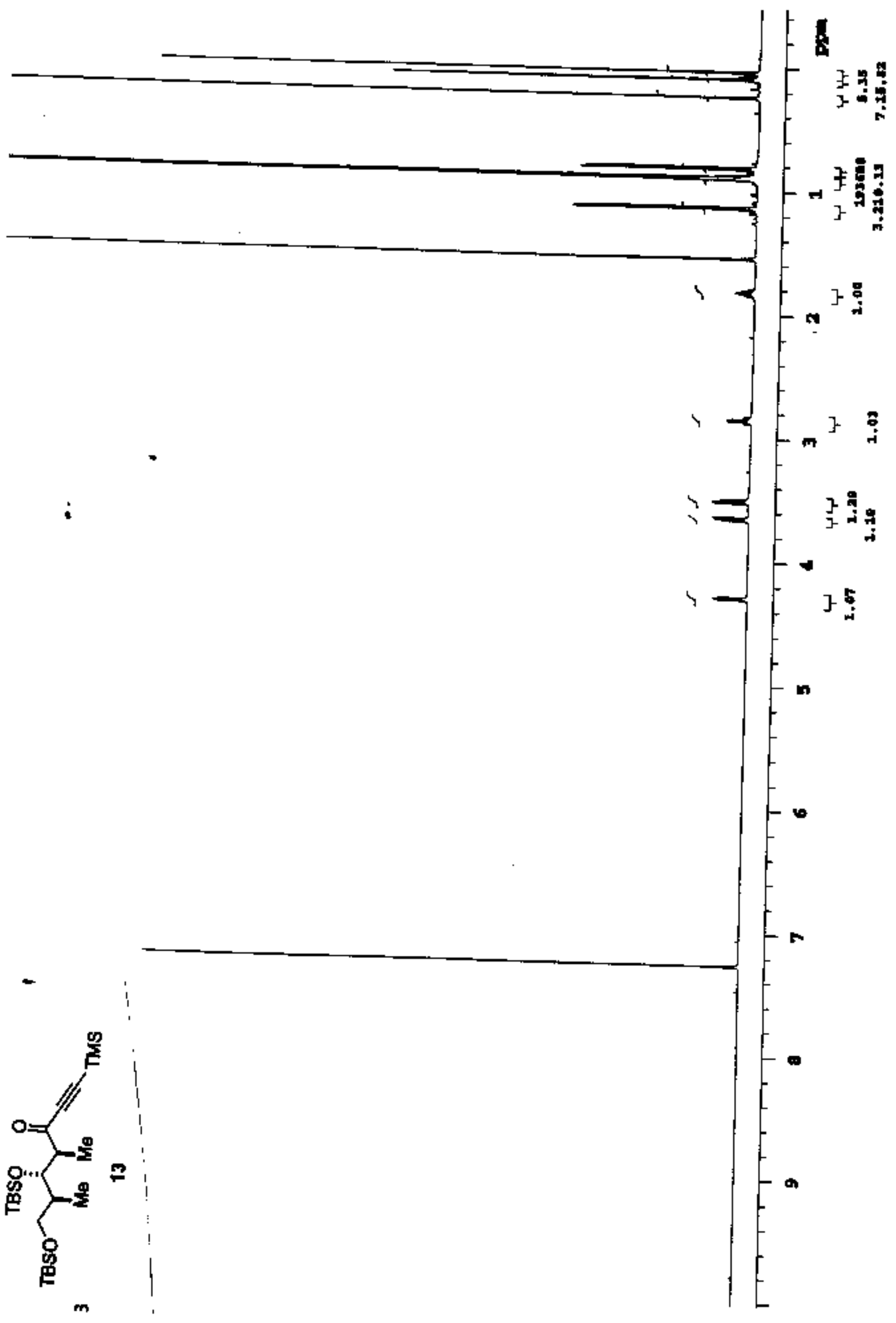




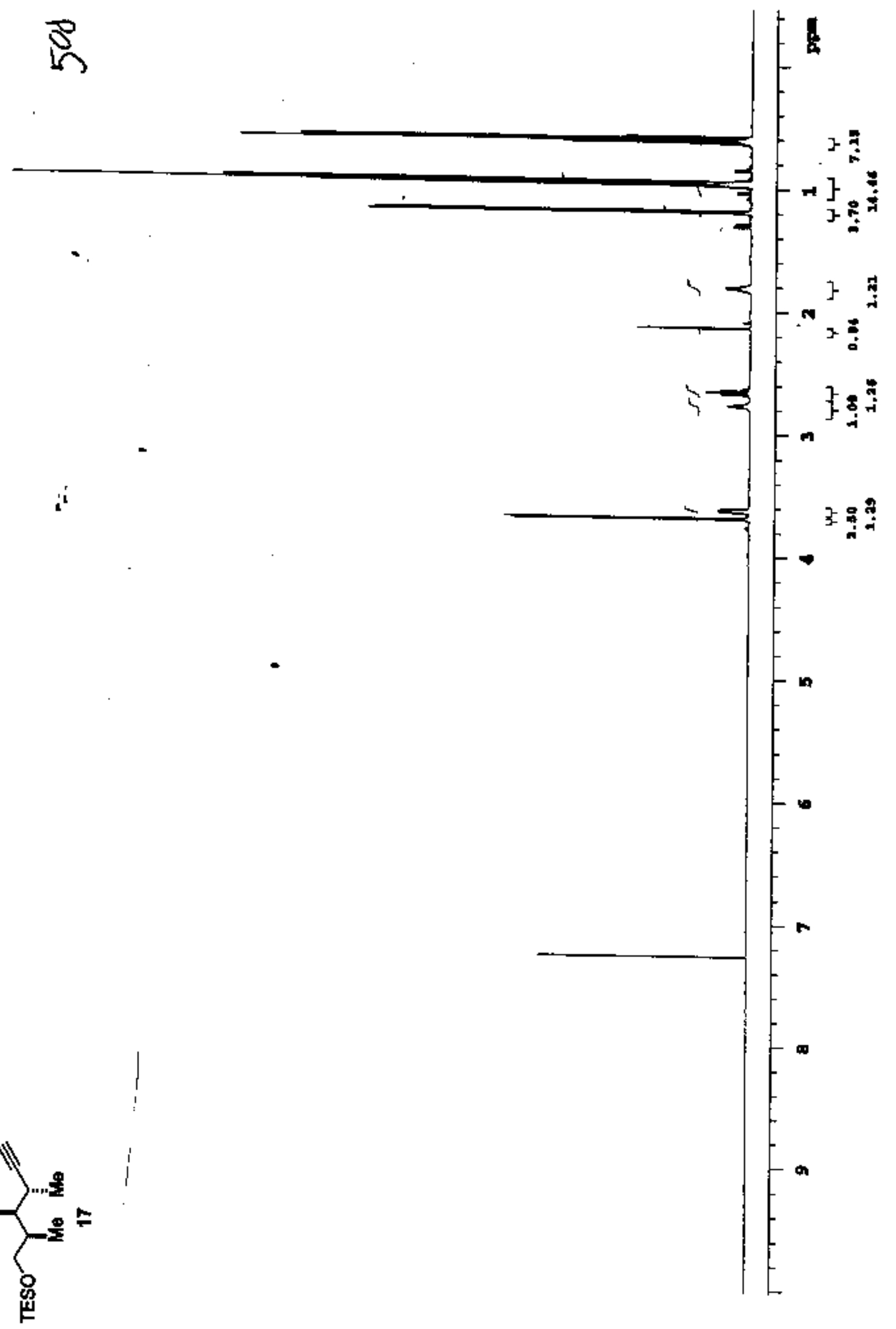




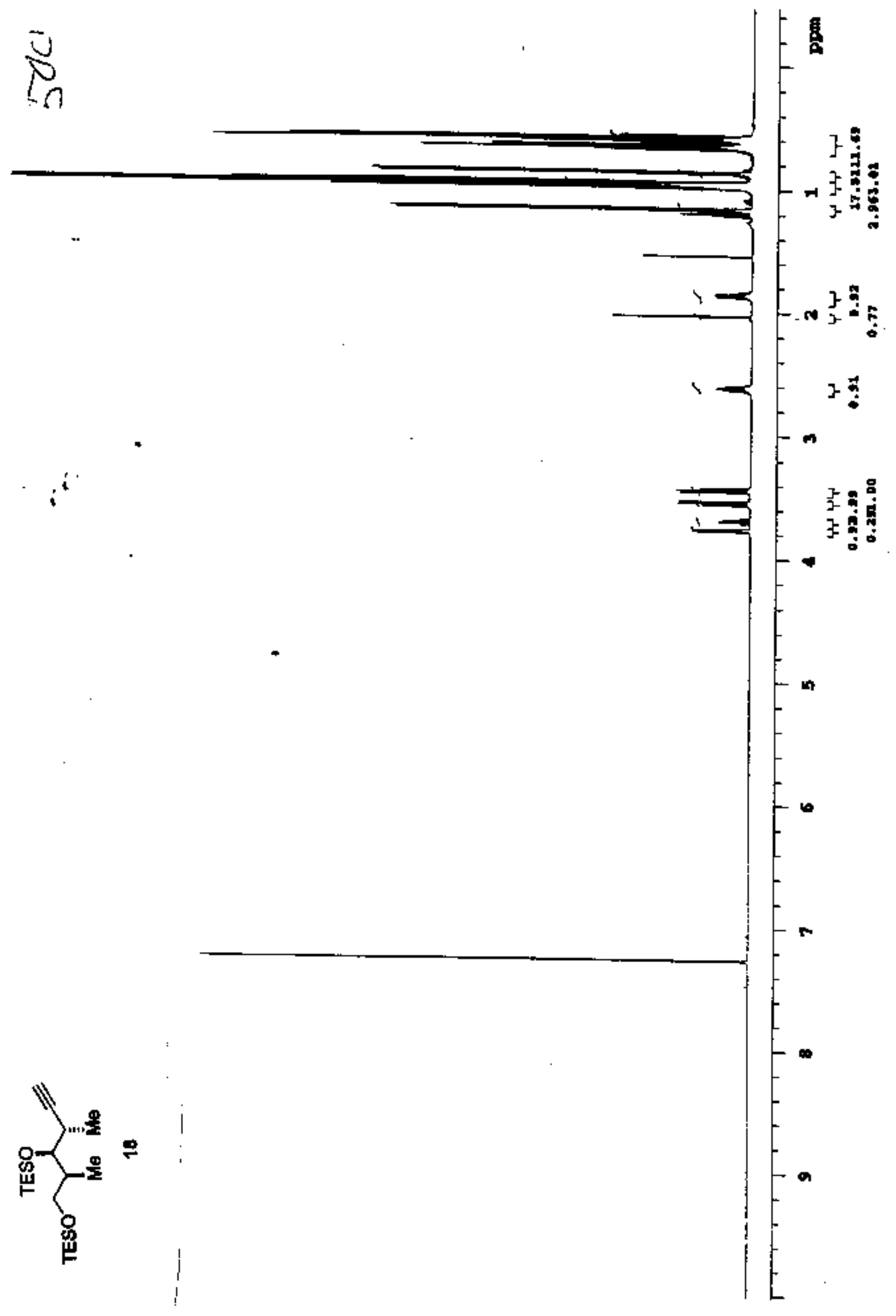




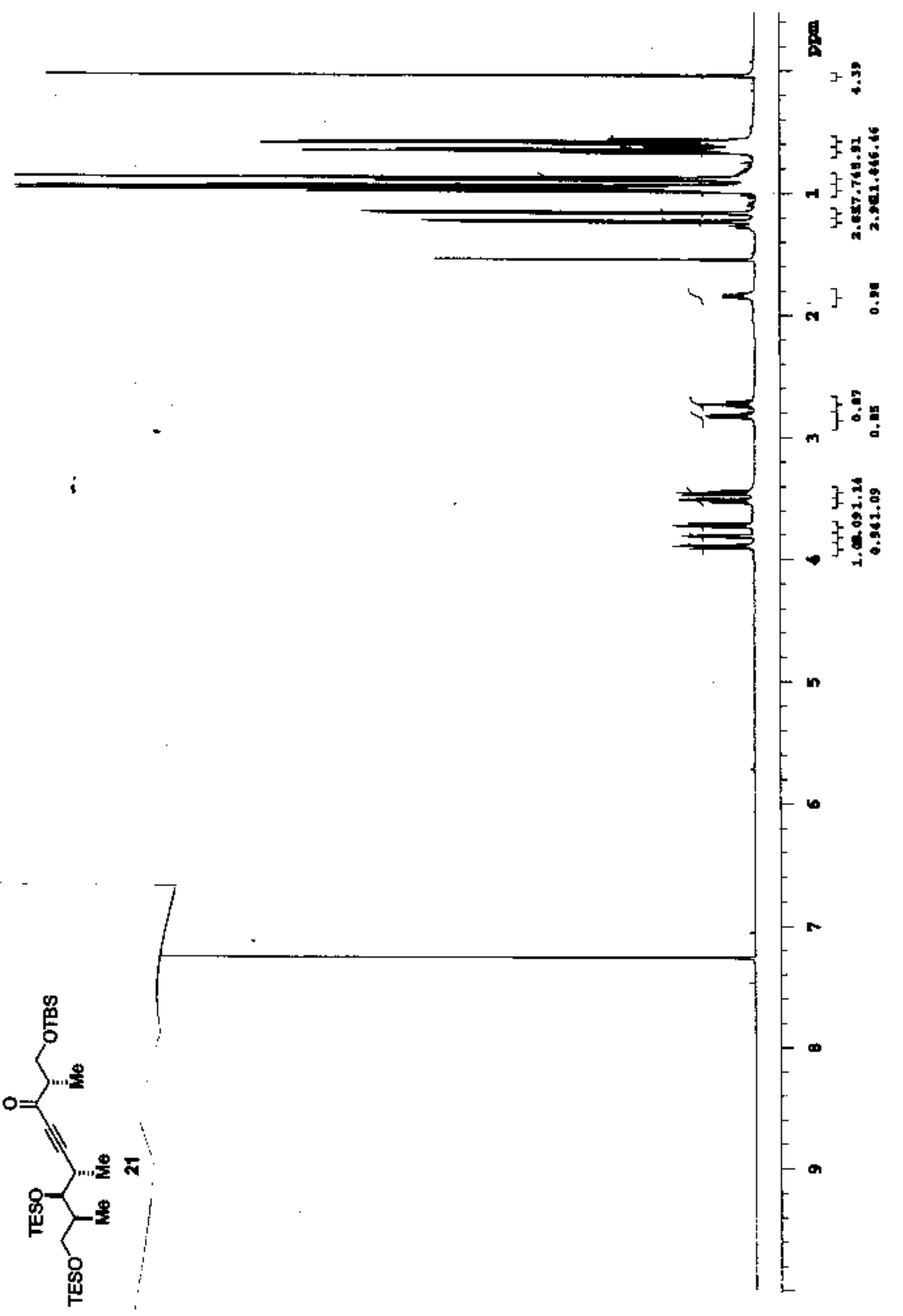


